\title{
A Stochastic Multiscale Method for the Elastodynamic Wave Equation Arising from Fiber Composites
}

\author{
Ivo Babuška ${ }^{\mathrm{a}}$, Mohammad Motamed $^{\mathrm{b}, *}$, Raúl Tempone ${ }^{\mathrm{c}}$ \\ ${ }^{a}$ Institute for Computational Engineering and Sciences, The University of Texas at \\ Austin, USA \\ ${ }^{b}$ Department of Mathematics and Statistics, The University of New Mexico, USA \\ ${ }^{c} S R I$ Center for Uncertainty Quantification in Computational Science and Engineering, \\ King Abdullah University of Science and Technology, Thuwal, Saudi Arabia
}

\begin{abstract}
We present a stochastic multilevel global-local algorithm for computing elastic waves propagating in fiber-reinforced composite materials. Here, the materials properties and the size and location of fibers may be random. The method aims at approximating statistical moments of some given quantities of interest, such as stresses, in regions of relatively small size, e.g. hot spots or zones that are deemed vulnerable to failure. For a fiber-reinforced cross-plied laminate, we introduce three problems (macro, meso, micro) corresponding to the three natural scales, namely the sizes of laminate, ply, and fiber. The algorithm uses the homogenized global solution to construct a good local approximation that captures the microscale features of the real solution. We perform numerical experiments to show the applicability and efficiency of the method.
\end{abstract}

Keywords: fiber composites, multiscale simulation, stochastic elastic wave equation, uncertainty quantification

\footnotetext{
*Corresponding author

Email addresses: babuska@ices.utexas.edu (Ivo Babuška), motamed@math.unm.edu (Mohammad Motamed), raul .tempone@kaust.edu.sa (Raúl Tempone)
} 


\section{Introduction}

Fiber-reinforced composite materials are used in various industrial products such as aerospace components (tails, wings, fuselages, propellers), automobiles, off-shore and marine structures, boats, magnetic resonance imaging (MRI) system components and many others.

Most fiber composites consist of stiff fibers in a matrix which is less stiff. The objective is to make a component which is strong and stiff. High stiffness and strength usually require a high proportion of fibers in the composite. This is achieved by aligning a set of long unidirectional fibers (with a diameter of approximately $5-10 \mu \mathrm{m}$ ) in a thin sheet (with a thickness of approximately 0.1-0.2 mm), called a lamina or ply. To achieve high strength and stiffness in various directions, a number of sheets are stacked and welded together, each having the fibers oriented in different directions. Such a stack is termed a cross-plied laminate, see Fig. 1.

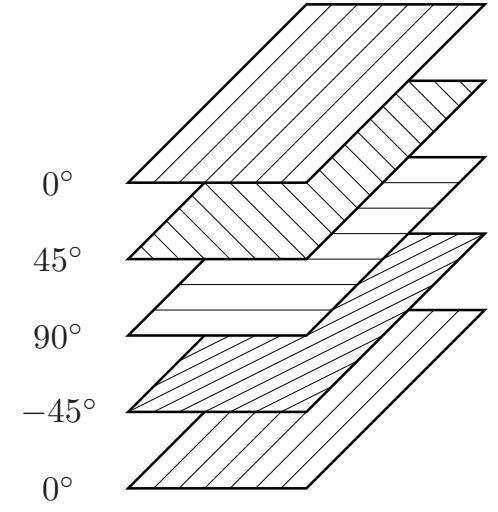

(a) A laminate of plies

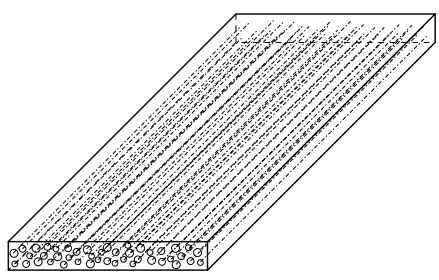

(b) An individual ply

Figure 1: (a) A composite laminate of plies with different angles of long unidirectional fibers. (b) One individual ply with unidirectional fibers with zero angle.

The long-term structural degradation of composite structures is largely influenced by micro mechanical events. The construction of a reliable method for predicting damage initiation and propagation has to be based on a multiscale approach. Moreover, due to the random character of the fiber locations and diameters, material properties, and fracture parameters, most mechanical quantities must be expressed in statistical terms, see [2].

The numerical approaches to multiscale problems are based on upscaling methods. A variety of numerical techniques have been proposed, including 
the numerical homogenization $[8,16]$, the generalized finite element method (GFEM) [1, 6, 3, 27], the variational multiscale method (VMS) [18], the heterogeneous multiscale methods (HMM) [12, 13, 15], and the multiscale finite element method (MsFEM) [17, 14].

In many engineering applications involving multiple scales, such as materials science, the main objective is to determine the local features of the fields, e.g. the maximum stresses, inside a small part of the domain. Such problems are amenable to global-local approaches, in which the homogenized global solution is used to construct a local solution that captures the micro scale features of the true multiscale solution. In such techniques, first a larger domain inside the global domain and containing the local domain is selected. Then, we employ a direct numerical method to solve the multiscale problem on this larger domain. The boundary conditions for the larger domain are obtained from the homogenized global solution, see e.g. $[24,28]$. We also refer to [4] which is based on the $L^{2}$-projection of the homogenized global solution onto function spaces spanned by solutions of local problems.

In this paper we consider a multiscale problem governed by the linear stochastic elastic wave equation arising from fiber composites. We are interested in the local features of the elastic field in regions of relatively small size, e.g. hot spots or zones that are deemed vulnerable to failure. Motivated and inspired by the work of [2], we assume that the diameter and position of fibers are random. The basic statistics of these parameters are taken from [2], which are obtained by carrying out a statistical analysis on a large set of data. Figure 2 shows a part of the composite plate consisting of 16275 fibers studied in [2].

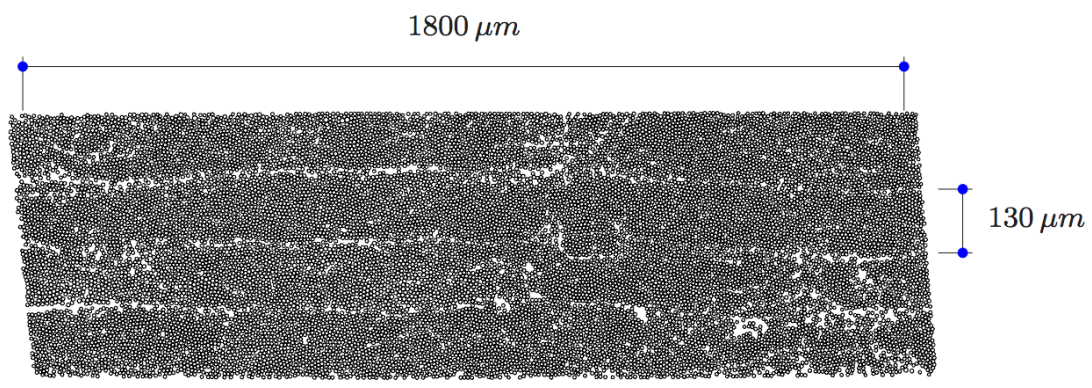

Figure 2: A group of four uni-directional plies consisting of 16275 fibers, taken from [2].

Corresponding to the three scales (laminate, ply, fiber), we introduce three levels (macro, meso, micro) and present a multilevel global-local ap- 
proach consisting of three problems (see Figure 3):

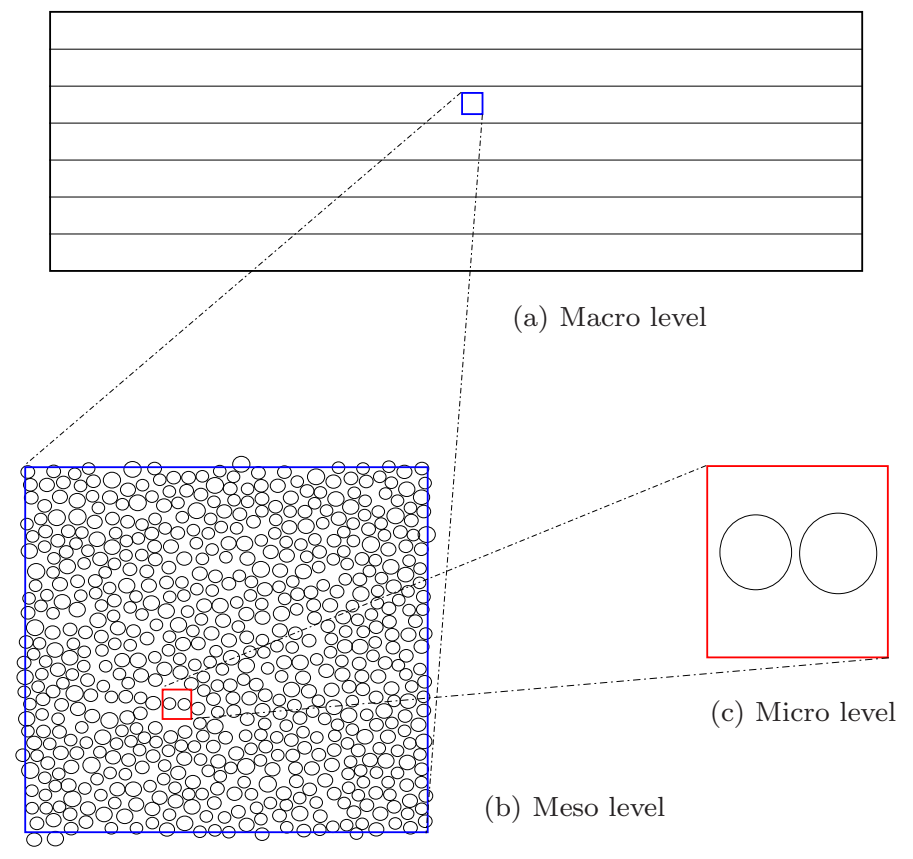

Figure 3: A schematic representation of different levels in the global-local multiscale method; (a) macro level in laminate scale, (b) meso level in ply scale, and (c) micro level in fiber scale.

I. Macro problem (Figure 3a). In this level, we construct a global solution on a corse grid based on the effective properties of the composite laminate. The effective macroscale or the homogenized material properties are obtained either by putting strain gages on the side of the laminate, or by employing stochastic homogenization techniques. Deterministic Dirichlet or Neumann boundary conditions are imposed on the boundaries of the laminate. Since the effective coefficients and boundary conditions are deterministic, we solve the deterministic elastic wave equation by finite element or finite difference methods. The global solution is then used to compute deterministic Dirichlet boundary data on the boundary of the meso problem.

II. Meso problem (Figure 3b). In this intermediate level, a domain (of ply thickness size) containing the small region of interest is first selected. The effective material properties are obtained by first applying deterministic homogenization techniques and then representing the homogenized coefficients as cross-correlated random fields with spatial correlations of ply thickness 
size. The intermediate solution is then used to compute stochastic Dirichlet boundary data on the boundary of the micro problem.

III. Micro problem (Figure 3c). Finally, in the micro level, the statistics of the quantity of interest in the local region of interest is computed. The stochastic material properties are directly given by the random location and diameter of fibers, represented in terms of a few random variables. A stochastic collocation method is employed to solve the resulting local stochastic elastic wave equation with stochastic boundary conditions.

The rest of the paper is organized as follows. In Section 2, we formulate the multiscale problem. In Section 3, we present and describe the multilevel global-local approach. A few notes on the error analysis of the multiscale method is made in Section 4. In Section 5, we perform numerical examples. Finally, we present our conclusions and future directions in Section 6 .

\section{Problem formulation}

Without loss of generality, we consider the case of plane strain in $\mathbb{R}^{3}$ and let $D$ be an open subset of $\mathbb{R}^{2}$ representing an orthogonal cross section of the laminate with boundary $\partial D$. Let $(\Omega, \mathcal{F}, P)$ be a complete probability space, where $\Omega$ is the set of outcomes, $\mathcal{F} \subset 2^{\Omega}$ is the $\sigma$-algebra of events and $P: \mathcal{F} \rightarrow[0,1]$ is a probability measure. The microscale problem is the following linear stochastic initial boundary value problem (IBVP) for anisotropic elastic materials: find a random vector-valued function $\mathbf{u}:[0, T] \times$ $\bar{D} \times \Omega \rightarrow \mathbb{R}^{2}$ such that $P$-almost everywhere in $\Omega$, i.e. almost surely (a.s), the following holds:

$$
\begin{array}{ll}
\varrho(\mathbf{x}, \omega) \mathbf{u}_{t t}(t, \mathbf{x}, \omega)-\nabla \cdot \boldsymbol{\sigma}(\mathbf{u}(t, \mathbf{x}, \omega))=\mathbf{f}(t, \mathbf{x}) & \text { in }[0, T] \times D \times \Omega, \\
\mathbf{u}(0, \mathbf{x}, \omega)=\mathbf{g}_{1}(\mathbf{x}), \quad \mathbf{u}_{t}(0, \mathbf{x}, \omega)=\mathbf{g}_{2}(\mathbf{x}) & \text { on }\{t=0\} \times D \times \Omega, \\
\mathbf{u}(t, \mathbf{x}, \omega)=\mathbf{h}_{d}(t, \mathbf{x}) & \text { on }[0, T] \times \partial D_{1} \times \Omega, \\
\boldsymbol{\sigma}(\mathbf{u}(t, \mathbf{x}, \omega)) \cdot \hat{\mathbf{n}}=\mathbf{h}_{n}(t, \mathbf{x}) & \text { on }[0, T] \times \partial D_{2} \times \Omega
\end{array}
$$

Here, $\varrho(\mathbf{x}, \omega)$ is the density, $\mathbf{u}=\left(u_{1}, u_{2}\right)^{\top}$ is the displacement vector, $t$ and $\mathbf{x}=\left(x_{1}, x_{2}\right)^{\top}$ are time and location, respectively, and $\boldsymbol{\sigma}$ is the stress tensor, whose components are given by the matrix form of Hook's law,

$\boldsymbol{\sigma}=\left[\begin{array}{cc}\sigma_{x_{1}} & \sigma_{x_{1} x_{2}} \\ \sigma_{x_{1} x_{2}} & \sigma_{x_{2}}\end{array}\right],\left[\begin{array}{c}\sigma_{x_{1}} \\ \sigma_{x_{2}} \\ \sigma_{x_{1} x_{2}}\end{array}\right]=C\left[\begin{array}{c}\varepsilon_{x_{1}} \\ \varepsilon_{x_{2}} \\ \varepsilon_{x_{1} x_{2}}\end{array}\right], C=\left[\begin{array}{ccc}C_{11} & C_{12} & C_{13} \\ C_{12} & C_{22} & C_{23} \\ C_{13} & C_{23} & C_{33}\end{array}\right]$ 
Here, $C(\mathbf{x}, \omega)$ is the material stiffness matrix, and $\varepsilon_{x_{1}}, \varepsilon_{x_{2}}$ and $\varepsilon_{x_{1} x_{2}}$ are the components of the strain tensor $\varepsilon$.

We augment the stochastic PDE (1a) with deterministic initial conditions (1b), and impose Dirichlet and Neumann conditions (1c)-(1d) on the nonoverlapping boundaries $\partial D_{1}$ and $\partial D_{2}$, respectively, where $\partial D=\partial D_{1} \cup \partial D_{2}$. The outward unit normal to the boundary is denoted by $\hat{\mathbf{n}}$.

The density $\varrho$ and the stiffness matrix $C$ are the main sources of randomness. They are assumed to be uniformly positive definite and bounded almost surely. This assumption will guarantee that the energy is conserved and therefore the stochastic IBVP (1) is well-posed [21]. Note that both the force term and boundary data may also be random. We also note that $\varrho$ and the components of the matrix $C$ may be correlated. In this work, however, for simplicity, we assume that only the components of $C$ are correlated, and $\varrho$ and $C$ are uncorrelated. The correlation between $\varrho$ and $C$ can be treated in the same way as the correlation between the components of $C$.

The density and stiffness matrix are directly obtained from the random location and diameter of fibers obtained by micrographs. Typically, in a laminate of cross sectional area $1 \mathrm{~mm}^{2}$, there are about 10,000 fibers. Due to the probabilistic character of the composite micro-structure and the large number of fibers, we encounter a multiscale problem, and a direct simulation is not feasible. We therefore propose a stochastic multiscale method that effectively captures the statistical microscale features of the real solution.

Remark 1. For isotropic materials, in the case of plane strain $\left(\varepsilon_{x_{3}}=0\right)$, the components of $C$ are given by

$$
\begin{aligned}
& C_{11}=C_{22}=\frac{E(1-\nu)}{(1+\nu)(1-2 \nu)}=\lambda+2 \mu, \\
& C_{12}=\frac{E \nu}{(1+\nu)(1-2 \nu)}=\lambda, \\
& C_{13}=C_{23}=0, \\
& C_{33}=\frac{E}{2(1+\nu)}=\mu,
\end{aligned}
$$

where, $E, \nu, \lambda$ and $\mu$ are the modulus of elasticity, poisson's ratio, and Lame's first and second parameters, respectively. Isotropic materials have therefore only two independent elastic parameters. In this case the stress tensor reads

$$
\boldsymbol{\sigma}(\mathbf{u})=\lambda(\mathbf{x}, \omega) \nabla \cdot \mathbf{u} I+\mu(\mathbf{x}, \omega)\left(\nabla \mathbf{u}+\nabla \mathbf{u}^{\top}\right) .
$$




\section{Stochastic multilevel global-local algorithm}

Let $D \subset \mathbb{R}^{2}$ be a global domain, which is an orthogonal cross-section of a laminate made of fiber-reinforced composite materials in the case of plane strain, and consider the IBVP (1). Moreover, assume that the materials properties and the size and position of fibers are given. Due to the random character of these given information, we first need to express the coefficients in (1) in statistical terms. We then want to compute the statistical moments of some quantities of interest, such as local displacements or stresses, in regions of relatively small size, e.g. hot spots or zones that are deemed vulnerable to failure. For this purpose, here we present and describe a multilevel global-local algorithm.

\subsection{Computational method}

First, corresponding to the three separate scales (laminate, ply, and fiber), we introduce and consider three levels/problems: macro or global; meso or intermediate; and micro or local. Consequently, we choose three computational domains corresponding to the three levels:

1. $D$ : the global domain which is the whole laminate.

2. $D^{I} \subset D$ : the intermediate domain of the ply thickness size and containing the local region of interest.

3. $D^{L} \subset D^{I}$ : the local domain which is the given small region of interest.

Next, for each domain, we compute the corresponding stiffness matrix, as described below:

1. Local stiffness matrix. The local stiffness matrix $C^{L}$ in $D^{L}$ is given by the actual size and position of fibers and the materials properties. For example, suppose that there are $N_{f}$ fibers in $D^{L}$. Each fiber is represented by three random variables: one for fiber's diameter and two for the location of fiber's center. Motivated by [2], we use truncated Gaussian variables for representing fibers' diameter. Moreover, we can use uniform random variables to represent the position of fibers' center. The random distributions are obtained from micrograph data. We also note that the intervals should be chosen so that the generated fibers do not overlap. In total, there are $N^{L}=3 N_{f}$ random variables giving a random vector $Y^{L}$ of $N_{f}$ independent truncated Gaussian and $2 N_{f}$ independent uniform variables. This generates the local random matrix $C^{L}=C^{L}\left(\mathrm{x}, Y^{L}\right)$ in $D^{L}$. 
2. Intermediate stiffness matrix. For the meso problem, we first follow [2] and cover $D^{I}$ by smaller square frames, each containing a few number of fibers. For example, consider an intermediate domain of size $400 \times 266.67 \mu \mathrm{m}$, studied in [2] and shown in Figure 4 . We cover it by $15 \times 10$ frames of side

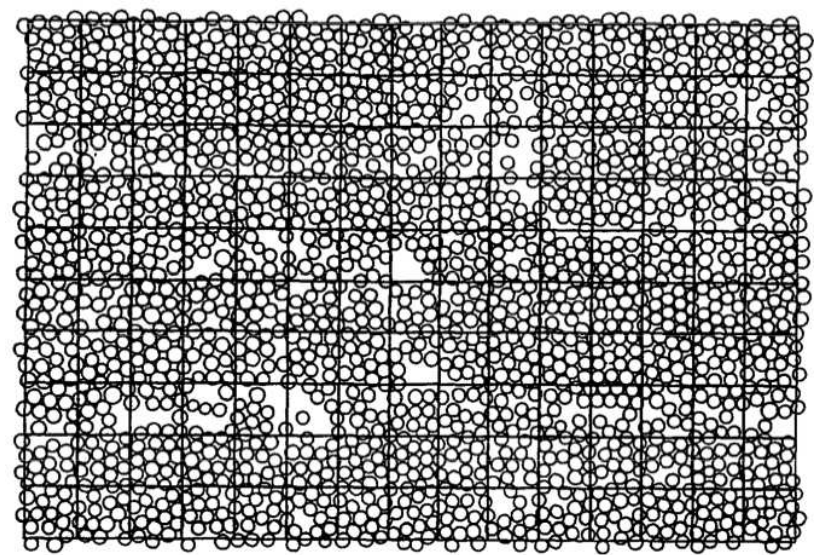

Figure 4: The intermediate domain studied in [2] and covered by $15 \times 10$ frames.

$26.67 \mu \mathrm{m}$. In each frame, we perform periodic homogenization to obtain effective coefficients. Let $\epsilon>0$ be a small parameter representing the ratio between the fiber and the ply length scales. Also assume that the exact multiscale coefficients $\lambda_{\epsilon}^{I}(\mathbf{x})$ and $\mu_{\epsilon}^{I}(\mathbf{x})$, described by the actual size and location of fibers in $D^{I}$, be of the form $\lambda^{I}(\mathbf{x} / \epsilon)$ and $\mu^{I}(\mathbf{x} / \epsilon)$, respectively. Since these coefficients are not periodic, by considering larger frames containing the small frames, we extend the coefficients periodically and apply the homogenization theory for periodic media. This approach aims at deriving the asymptotic behavior of the multiscale solution $\mathbf{u}_{\epsilon}^{I}$ as $\epsilon \rightarrow 0$. The multiscale solution is first expanded in power series of $\epsilon$ :

$$
\mathbf{u}_{\epsilon}^{I}(t, \mathbf{x})=\mathbf{u}_{0}^{I}(t, \mathbf{x}, \mathbf{x} / \epsilon)+\epsilon \mathbf{u}_{1}^{I}(t, \mathbf{x}, \mathbf{x} / \epsilon)+\epsilon^{2} \mathbf{u}_{2}^{I}(t, \mathbf{x}, \mathbf{x} / \epsilon)+\ldots
$$

By plugging the expansion into the governing equations for the meso problem and equating like powers of $\epsilon$, a time-independent cell problem with periodic boundary conditions is obtained from which the homogenized coefficients, $\lambda^{H}$ and $\mu^{H}$, are computed. See e.g. [2, 7] for more details.

The homogenized Lame parameters, $\lambda^{H}$ and $\mu^{H}$, computed by periodic homogenization and based on the Lame parameters given in Table 1, are tabulated in Tables 2 and 3. Note that here, the Lame parameters $\lambda$ and $\mu$ 
of fibers and the matrix are assumed to be deterministic, and the randomness is only due to the random spatial distribution and random size of fibers. This is however not a restrictive assumption, and the case when $\lambda$ and $\mu$ of fibers and the matrix are also random can be easily treated.

Table 1: Lame parameters for the composite under consideration.

\begin{tabular}{ccc}
\hline composite phases & $\lambda$ & $\mu$ \\
\hline fiber & 8.9330 & 9.6774 \\
matrix & 2.0769 & 1.3846 \\
\hline
\end{tabular}

Table 2: Effective Lame's first parameter $\lambda^{H}$ from the $15 \times 10$ grid shown in Figure 4 .

\begin{tabular}{lllllllllllllll}
\hline 4.9 & 3.4 & 4.2 & 3.7 & 4.2 & 5.1 & 3.7 & 4.4 & 3.6 & 4.0 & 3.4 & 2.6 & 3.1 & 4.1 & 4.0 \\
3.4 & 3.7 & 4.8 & 4.2 & 4.6 & 4.4 & 4.2 & 4.9 & 2.1 & 3.0 & 3.4 & 4.9 & 4.3 & 3.3 & 3.7 \\
3.1 & 3.6 & 4.2 & 4.5 & 4.7 & 4.2 & 4.5 & 3.2 & 4.5 & 2.6 & 4.5 & 4.0 & 4.1 & 4.8 & 3.3 \\
3.4 & 3.2 & 4.2 & 4.5 & 3.9 & 3.2 & 4.8 & 4.3 & 4.9 & 3.8 & 3.8 & 3.5 & 3.4 & 3.9 & 3.3 \\
4.8 & 4.4 & 2.7 & 3.5 & 3.2 & 3.8 & 3.6 & 3.3 & 4.5 & 3.5 & 4.8 & 3.7 & 3.1 & 3.0 & 5.1 \\
4.5 & 3.2 & 4.3 & 3.5 & 4.7 & 3.8 & 2.4 & 4.0 & 3.6 & 5.5 & 4.3 & 4.1 & 4.9 & 3.6 & 4.0 \\
3.4 & 4.3 & 4.0 & 3.8 & 2.3 & 3.9 & 3.8 & 2.6 & 4.0 & 4.7 & 5.2 & 3.9 & 4.9 & 4.0 & 4.2 \\
5.2 & 4.8 & 3.7 & 3.1 & 4.2 & 2.3 & 4.4 & 5.7 & 4.0 & 4.2 & 3.6 & 4.2 & 4.2 & 4.7 & 3.9 \\
3.2 & 3.9 & 4.4 & 5.0 & 2.6 & 4.7 & 3.3 & 5.1 & 3.8 & 3.9 & 3.6 & 4.1 & 3.3 & 3.8 & 4.9 \\
4.0 & 3.3 & 3.6 & 3.9 & 4.2 & 3.8 & 4.2 & 3.2 & 4.0 & 3.0 & 3.9 & 3.2 & 4.5 & 4.2 & 3.3 \\
\hline
\end{tabular}

Table 3: Effective Lame's second parameter $\mu^{H}$ from the $15 \times 10$ grid shown in Figure 4 .

\begin{tabular}{lllllllllllllll}
\hline 4.6 & 4.2 & 4.5 & 4.4 & 4.0 & 4.1 & 3.9 & 4.0 & 2.6 & 3.2 & 3.5 & 3.4 & 3.9 & 4.2 & 3.5 \\
3.8 & 4.2 & 4.5 & 4.3 & 4.6 & 4.5 & 4.2 & 4.4 & 2.4 & 2.5 & 4.1 & 4.8 & 4.2 & 3.1 & 3.3 \\
2.7 & 2.5 & 3.4 & 3.9 & 3.8 & 3.4 & 3.5 & 2.7 & 3.2 & 1.8 & 3.9 & 3.8 & 4.1 & 3.9 & 3.3 \\
4.0 & 3.5 & 4.1 & 3.9 & 4.1 & 3.8 & 4.5 & 4.0 & 4.6 & 3.0 & 4.3 & 4.1 & 3.7 & 4.2 & 3.3 \\
4.4 & 4.1 & 3.3 & 2.6 & 2.8 & 3.0 & 3.9 & 2.8 & 3.8 & 2.8 & 4.8 & 3.9 & 4.1 & 3.3 & 3.9 \\
3.9 & 3.5 & 4.4 & 3.8 & 4.6 & 3.8 & 4.0 & 3.4 & 3.9 & 4.5 & 4.1 & 4.9 & 5.0 & 3.6 & 4.3 \\
3.6 & 4.0 & 5.1 & 3.7 & 3.6 & 3.8 & 4.6 & 2.9 & 4.0 & 4.7 & 4.8 & 4.3 & 4.8 & 4.0 & 4.8 \\
4.3 & 3.7 & 2.3 & 2.2 & 2.9 & 2.1 & 3.7 & 3.6 & 3.3 & 3.2 & 2.6 & 2.7 & 3.3 & 3.1 & 3.3 \\
3.9 & 4.3 & 4.7 & 4.5 & 3.6 & 4.2 & 4.7 & 4.3 & 3.4 & 3.8 & 3.3 & 4.5 & 3.3 & 3.6 & 4.3 \\
4.2 & 3.9 & 4.9 & 4.5 & 3.7 & 3.7 & 4.5 & 3.8 & 3.9 & 2.9 & 3.6 & 3.7 & 4.3 & 4.6 & 4.4 \\
\hline
\end{tabular}


Next, we collect the basic statistical summaries of the effective parameters, given in Table 4.

Table 4: Statistical summaries of the effective homogenized Lame parameters for the $15 \times 10$ grid shown in Figure 4 .

\begin{tabular}{lll}
\hline Statistic & $\lambda^{H}$ & $\mu^{H}$ \\
\hline Min & 2.1 & 1.8 \\
Max & 5.8 & 5.1 \\
Mean & 3.9 & 3.7 \\
S.D. & 0.7 & 0.7 \\
\hline
\end{tabular}

This will be the basis for constructing empirical random fields $\lambda^{I}(\mathbf{x}, \omega)$ and $\mu^{I}(\mathbf{x}, \omega)$. Following [2], we may assume that the random fields $\lambda^{I}$ and $\mu^{I}$ have a stationary Gaussian covariance structure. For instance, the stationarity of the covariance structure is justified by Table 16 on page 69 of [2], which shows that the statistics in three different large frames of size $D^{I}$ are the same. We then obtain their spatial correlation lengths, say $L_{c}$, from the homogenized data $\lambda^{H}$ and $\mu^{H}$, see e.g. [19]. Moreover, the statistical analysis in [2] suggests that the random fields $\lambda^{I}$ and $\mu^{I}$ may also be cross-correlated with a cross correlation matrix

$$
C_{\mathrm{cross}}=\left[\begin{array}{ll}
1 & c \\
c & 1
\end{array}\right] .
$$

We then perform a truncated Karhunen-Loéve decomposition of cross correlated normal random fields [20] and obtain the effective random fields $G_{\lambda}\left(\mathbf{x}, Y_{\lambda}^{I}\right)$ and $G_{\mu}\left(\mathbf{x}, Y_{\mu}^{I}\right)$, where $Y^{I}=\left[Y_{\lambda}^{I}, Y_{\mu}^{I}\right]$ is a random vector of $N^{I}$ independent normal random variables. The number of modes $N^{I}$ in the Karhunen-Loéve expansion is chosen such that a high percentage of the standard deviation is preserved. Finally, in order for the empirical random fields $\lambda^{I}$ and $\mu^{I}$ to have correct marginal distributions, we let each random field have a beta distribution with four parameters: the exponents $\alpha$ and $\beta$, and the minimum and the maximum of the distribution range $a$ and $b$. These four parameters are computed so that the statistic measures in Table 4 are satisfied. For example, let $F_{\lambda}$ and $F_{\mu}$ be the resulting cumulative distribution 
functions (CDFs) corresponding to the statistical summaries of $\lambda^{H}$ and $\mu^{H}$, respectively. The random fields $\lambda^{I}$ and $\mu^{I}$ are then given by

$$
\lambda^{I}\left(\mathbf{x}, Y_{\lambda}^{I}\right)=F_{\lambda}^{-1} \circ \Phi\left(G_{\lambda}\left(\mathbf{x}, Y_{\lambda}^{I}\right)\right), \quad \mu^{I}\left(\mathbf{x}, Y_{\mu}^{I}\right)=F_{\mu}^{-1} \circ \Phi\left(G_{\mu}\left(\mathbf{x}, Y_{\mu}^{I}\right)\right),
$$

where $\Phi$ is the normal CDF. The stiffness matrix for the meso problem $C^{I}=$ $C^{I}\left(\mathbf{x}, Y^{I}\right)$ is therefore obtained in terms of the random vector $Y^{I}=\left[Y_{\lambda}^{I}, Y_{\mu}^{I}\right]$ consisting of $N^{I}$ independent normal random variables.

We note that the accuracy of empirical $\lambda^{I}$ and $\mu^{I}$, in addition to the Gaussian covariance and beta distribution assumptions, depends also on other parameters, such as their spatial correlation lengths $L_{c}$ and their crosscorrelation matrix $C_{\text {cross }}$. Therefore, in order for $\lambda^{I}$ and $\mu^{I}$ to have CDFs close to the CDFs of the homogenized coefficients $\lambda^{H}$ and $\mu^{H}$, the parameters need to be carefully selected based on calibration techniques. This is beyond the scope of the present paper and is the subject of our current work, which will be presented elsewhere. Here, in the numerical examples in Section 5, we choose the parameters $L_{c}$ and $C_{\text {cross }}$ simply based on the behavior of the homogenized coefficients in Tables 2 and 3. Despite this, the obtained CDFs of empirical and homogenized coefficients are in good agreement.

3. Global stiffness matrix. The effective global stiffness matrix $C^{G}$ in $D$ is a constant and deterministic matrix obtained either by putting strain gages on the side of the laminate, or by employing stochastic homogenization techniques under proper stationarity and ergodicity assumptions on the random fields obtained in the meso level. In such techniques, a realization of the random field on $D^{I}$ is first extended periodically to a larger finite domain $D_{\gamma}=[0, \gamma]^{2}$ containing $D^{I}$, with $\gamma>0$. Then periodic homogenization is employed to obtain effective global coefficients. See $[9,10,7]$ for further details on stochastic homogenization.

After computing stiffness matrices for the three problems, in the last step of the algorithm, we solve the following three problems simultaneously:

I. Micro problem. The main problem we want to solve is the micro problem in the local domain $D^{L}$,

$$
\begin{array}{ll}
\varrho\left(\mathbf{x}, Y^{L}\right) \mathbf{u}_{t t}(t, \mathbf{x}, Y)-\nabla \cdot \boldsymbol{\sigma}(\mathbf{u}(t, \mathbf{x}, Y))=\mathbf{f} & \text { in }[0, T] \times D^{L} \times \Gamma \\
\mathbf{u}(0, \mathbf{x}, Y)=\mathbf{g}_{1}(\mathbf{x}), \quad \mathbf{u}_{t}(0, \mathbf{x}, Y)=\mathbf{g}_{2}(\mathbf{x}) & \text { on }\{t=0\} \times D^{L} \times \Gamma, \\
\mathbf{u}(t, \mathbf{x}, Y)=\mathbf{g}^{I}\left(t, \mathbf{x}, Y^{I}\right) & \text { on }[0, T] \times \partial D^{L} \times \Gamma
\end{array}
$$

The random vector $Y=\left[Y^{L}, Y^{I}\right]$ has in total $N=N^{L}+N^{I}$ independent random variables $\left\{Y_{n}\right\}_{n=1}^{N}$. We denote by $\Gamma_{n} \equiv Y_{n}(\Omega)$ the image of $Y_{n}$ and let 
$\Gamma=\prod_{n=1}^{N} \Gamma_{n}$. We further assume that the random vector $Y \in \Gamma \subset \mathbb{R}^{N}$ has a bounded joint probability density function $\rho: \Gamma \rightarrow \mathbb{R}_{+}$with $\rho \in L^{\infty}(\Gamma)$. The random density $\varrho\left(\mathbf{x}, Y^{L}\right)$ and stiffness matrix $C^{L}\left(\mathbf{x}, Y^{L}\right)$ are directly obtained from the fiber positions and diameters, as described above. We use the stochastic collocation method (see Section 3.3) to compute the statistical moments of given quantities of interest. The boundary force term $\mathbf{g}^{I}\left(t, \mathbf{x}, Y^{I}\right)$ on $\partial D^{L}$ is obtained from the intermediate solution $\mathbf{u}^{I}$ to the following meso problem (see Section 3.2).

II. Meso problem. At each collocation point obtained in the micro problem, we solve the following problem in the intermediate domain $D^{I}$,

$$
\begin{array}{ll}
\varrho\left(\mathbf{x}, Y^{I}\right) \mathbf{u}_{t t}\left(t, \mathbf{x}, Y^{I}\right)-\nabla \cdot \boldsymbol{\sigma}\left(\mathbf{u}\left(t, \mathbf{x}, Y^{I}\right)\right)=\mathbf{f} & \text { in }[0, T] \times D^{I} \times \Gamma^{I}, \\
\mathbf{u}\left(0, \mathbf{x}, Y^{I}\right)=\mathbf{g}_{1}(\mathbf{x}), \quad \mathbf{u}_{t}\left(0, \mathbf{x}, Y^{I}\right)=\mathbf{g}_{2}(\mathbf{x}) & \text { on }\{t=0\} \times D^{I} \times \Gamma^{I}, \\
\mathbf{u}\left(t, \mathbf{x}, Y^{I}\right)=\mathbf{g}^{G}(t, \mathbf{x}) & \text { on }[0, T] \times \partial D^{I} \times \Gamma^{I},
\end{array}
$$

where the random density $\varrho\left(\mathbf{x}, Y^{I}\right)$ and stiffness matrix $C^{I}\left(\mathbf{x}, Y^{I}\right)$ are obtained based on $N^{I}$ independent Gaussian random variables, as described above, and $\Gamma^{I}=\prod_{n=N^{L}+1}^{N} \Gamma_{n}$. The boundary force term $\mathbf{g}^{G}(t, \mathbf{x})$ on $\partial D^{I}$ is obtained from the global solution $\mathbf{u}^{G}$ to the following macro problem.

III. Macro problem. The global solution $\mathbf{u}^{G}$ is obtained by solving the deterministic macro problem (1) in $D$ where the random density $\varrho$ and stiffness matrix $C$ are replaced with the effective deterministic constant density $\rho^{G}$ and constant stiffness matrix $C^{G}$. We use a deterministic solver such as the finite difference or the finite element method.

\subsection{Computation of internal boundary conditions}

Suppose that a two-dimensional local domain of interest $D^{L} \subset D^{I}$ is given. The exact Dirichlet boundary condition on the boundary $\partial D^{L}$ reads

$$
\left.\mathbf{u}\right|_{\partial D^{L}}=\mathbf{g}^{*}\left(t, \mathbf{x}, Y^{I}\right), \quad \forall \mathbf{x} \in \partial D^{L} .
$$

For every fixed $Y^{I} \in \Gamma^{I}$, we can approximate the function $\mathbf{g}^{*}$ by the value of the intermediate solution $\mathbf{u}^{I}$ on $\partial D^{L}$ :

$$
\left.\mathbf{g}^{*}\left(t, \mathbf{x}, Y^{I}\right) \approx \mathbf{u}^{I}\left(t, \mathbf{x}, Y^{I}\right)\right|_{\partial D^{L}}=: \mathbf{g}^{I} .
$$

A simple way to do this is to compute $\mathbf{u}^{I}$ and its spatial derivatives $\partial_{x_{1}} \mathbf{u}^{I}$ and $\partial_{x_{2}} \mathbf{u}^{I}$ at the center $\mathbf{x}_{c}$ of the domain $D^{L}$ and then apply Taylor expansion around this point. We obtain for every $\mathbf{x} \in \partial D^{L}$,

$$
\left.\left.u_{i}^{I}\right|_{\partial D^{L}} \approx u_{i}^{I}\right|_{\mathbf{x}_{c}}+\left.\left(\mathbf{x}-\mathbf{x}_{c}\right)^{\top} \nabla u_{i}^{I}\right|_{\mathbf{x}_{c}}, \quad i=1,2 .
$$


In a similar way, we can obtain the boundary condition for the intermediate problem.

Remark 2. It is well known that this approximation introduces the so-called pollution effects $[24,28]$. One way to reduce the error is to consider a larger domain containing $D^{L}$. We can further improve the accuracy of (6) by adding correction terms. We write

$$
\mathbf{g}^{*} \approx \mathbf{g}^{I}=\left(g_{1}, g_{2}\right)^{\top}, \quad g_{i}=\left.u_{i}^{I}\right|_{\partial D^{L}}+\left.\varphi^{\top} \nabla u_{i}^{I}\right|_{\partial D^{L}}, \quad i=1,2 .
$$

Here, $u_{i}^{I}$ and $\nabla u_{i}^{I}$ on $\partial D^{L}$ are computed from (7). Moreover, to compute $\boldsymbol{\varphi}$, we first notice that the solution on the boundary given in (8) needs to satisfy the PDE in the micro problem. This gives a PDE for $\varphi$. We augment the PDE with periodic boundary conditions.

\subsection{Stochastic collocation method}

In this section, we briefly review the stochastic collocation method for computing the statistical moments of the solution to the micro problem (4), where $Y \in \Gamma \subset \mathbb{R}^{N}$ is a vector of $N$ independent random variables [5, 29, 22].

The stochastic collocation method consists of three main steps. First, the problem (4) is discretized in space and time, using a deterministic numerical method, such as the finite element or the finite difference method. We denote the semi-discrete solution by $\mathbf{u}_{h}(t, \mathbf{x}, Y)$, where $h$ represents the discretization mesh size and time step. The obtained semi-discrete problem is then collocated in a set of $\eta$ collocation points $\left\{Y^{(k)}\right\}_{k=1}^{\eta} \in \Gamma$ to compute the approximate solutions $\mathbf{u}_{h}\left(t, \mathbf{x}, Y^{(k)}\right)$. Finally, a global polynomial approximation $\mathbf{u}_{h, p}$ is built upon those evaluations

$$
\mathbf{u}_{h, p}(t, \mathbf{x}, Y)=\sum_{k=1}^{\eta} \mathbf{u}_{h}\left(t, \mathbf{x}, Y^{(k)}\right) L_{k}(Y),
$$

for suitable multivariate polynomials $\left\{L_{k}\right\}_{k=1}^{\eta}$ such as Lagrange polynomials. Here, $p$ represents the polynomial degree.

A key point in the stochastic collocation method is the choice of the set of collocation points $\left\{Y^{(k)}\right\}$, i.e. the type of computational grid in the $N$ dimensional stochastic space. A full tensor grid, based on cartesian product of mono-dimensional grids, can only be used when the number of stochastic dimensions $N$ is small, since the computational cost grows exponentially fast with $N$ (curse of dimensionality). 
Alternatively, sparse grids can reduce the curse of dimensionality. They were originally introduced by Smolyak for high dimensional quadrature and interpolation computations [25]. Two typical examples of sparse grids include total degree and hyperbolic cross sparse grids. We refer to [5, 29, 22] for more details.

The ultimate goal of the computations is the prediction of statistical moments of some given quantities of interest $\mathcal{Q}(\mathbf{u})$. The quantity of interest may be either a function, e.g. the solution $\mathbf{u}$, or a functional of the solution, e.g. the spatial and temporal averages of the solution, or an operator applied to the solution, e.g. the components of the stress tensor. Using the Gauss quadrature formula for approximating integrals, we write

$$
\begin{aligned}
\mathbb{E}[\mathcal{Q}(\mathbf{u}(., Y))] & \approx \mathbb{E}\left[\mathcal{Q}\left(\mathbf{u}_{h}(., Y)\right)\right]=\int_{\Gamma} \mathcal{Q}\left(\mathbf{u}_{h}(., Y)\right) \rho(Y) d Y \\
& \approx \sum_{k=1}^{\eta(\ell)} \theta_{k} \mathcal{Q}\left(\mathbf{u}_{h}\left(., Y^{(k)}\right)\right)=: \mathbb{E}_{\ell}\left[\mathcal{Q}\left(\mathbf{u}_{h}(., Y)\right)\right]
\end{aligned}
$$

The weights are

$$
\theta_{k}=\prod_{n=1}^{N} \int_{\Gamma_{n}} L_{k_{n}}\left(Y_{n}\right) \rho_{n}\left(Y_{n}\right) d Y_{n}, \quad L_{k_{n}}\left(Y_{n}\right)=\prod_{i=0, i \neq k_{n}}^{\eta} \frac{Y_{n}-Y_{n}^{(i)}}{Y_{n}^{\left(k_{n}\right)}-Y_{n}^{(i)}}
$$

and the collocation points $Y^{(k)}=\left[Y_{1}^{\left(k_{1}\right)}, \ldots, Y_{N}^{\left(k_{N}\right)}\right] \in \Gamma$ are tensorized Gauss points with $Y_{n}^{\left(k_{n}\right)}, k_{n}=0,1, \ldots, p_{n}$, being the zeros of the $\rho_{n}$-orthogonal polynomial of degree $p_{n}+1$. Here, for any vector of indices $\left[k_{1}, \ldots, k_{N}\right]$ with $0 \leq k_{n} \leq p_{n}$ the associated global index reads $k=1+k_{1}+\left(p_{1}+1\right) k_{2}+\left(p_{1}+\right.$ 1) $\left(p_{2}+1\right) k_{3}+\ldots$ The positive integer $\ell$ is called the level, and $p_{n}(\ell)$ is the maximum degree of polynomials in the $n$-th direction, with $n=1, \ldots, N$, given as a function of the level $\ell$.

\subsection{Stochastic regularity of quantities of interest}

The convergence rate of error in the stochastic collocation method depends on the regularity of the quantity of interest $\mathcal{Q}\left(\mathbf{u}_{h}\right)$ with respect to the random input variable $Y$. This regularity is called stochastic regularity, which is in turn related to the regularity of the coefficients and data in the physical space. A fast/exponential convergence is obtained in the presence of high stochastic regularity or stochastic analyticity. 
Recently, it is shown in $[22,21]$ that the solution of stochastic hyperbolic problems, unlike the solution of elliptic and parabolic problems, is not in general analytic with respect to the random variables. The wave solutions may posses high regularity for particular types of smooth data. However, in real applications, the data are not smooth. Therefore, the convergence rate of error in the wave solution may only be algebraic. Yet, a fast spectral convergence is possible for mollified quantities of interest.

Following [21], we propose to perform a low-pass filtering (moving average) in order to isolate and remove the non-physical oscillations in $\mathcal{Q}\left(\mathbf{u}_{h}\right)$, which are a result of its low regularity. This is done by convolving the oscillatory quantity of interest with a Gaussian kernel

$$
K_{\delta}(\mathbf{x})=\frac{1}{2 \pi \delta^{2}} \exp \left(-\frac{|\mathbf{x}|^{2}}{2 \delta^{2}}\right),
$$

with the standard deviation $\delta$. See Remark 3 for more comments on $\delta$. The filtered quantity is then given by

$$
\mathcal{Q}_{\delta}\left(\mathbf{u}_{h}\right)(t, \mathbf{x}, Y)=\left(\mathcal{Q}\left(\mathbf{u}_{h}\right) \star K_{\delta}\right)(t, \mathbf{x}, Y)=\int_{D^{L}} K_{\delta}(\mathbf{x}-\tilde{\mathbf{x}}) \mathcal{Q}\left(\mathbf{u}_{h}\right)(t, \tilde{\mathbf{x}}, Y) d \tilde{\mathbf{x}}
$$

We note that due to the boundary effects introduced by the convolution, we compute the filtered quantity on a smaller domain $\mathbf{x} \in D_{\delta} \subset D^{L}$ with $\operatorname{dist}\left(\partial D_{\delta}, \partial D^{L}\right) \geq d>0$. We choose $d$ so that $K_{\delta}\left(\mathbf{x}_{0}\right)$ with $\left|\mathbf{x}_{0}\right|=d$ is essentially zero. This implies that for any $\mathbf{x} \in D_{\delta}$, the support of $K_{\delta}(\mathbf{x}-\tilde{\mathbf{x}})$ is essentially vanishing at $\partial D^{L}$. A practical choice is for example $d \geq 2 \delta$.

Remark 3. There are two different motivations for performing a low-pass filter; physical and numerical ones. The physical motivation arises for instance in seismology, where the simulated seismic data are often post-processed by performing a low-pass filter in order to isolate and remove the high-frequency modes. An example is when high-frequency modes do not excite a given infrastructure and are therefore removed. The numerical motivation arises for instance due to the presence of high-frequency modes which are not resolved on the spatial mesh. In this case, the generated high-frequency noise in the solution is filtered out for accuracy reasons. Another example is when we require higher regularity in the solution with respect to a given input parameter, which is obtained by performing a low-pass filter. We note that when the filtered solution is a physical quantity, the standard deviation $\delta$ is a given 
constant. In fact, in the example mentioned here, $\delta$ is inversely proportional to the maximum frequency that is allowed to pass. On the other hand, when a low-pass filter is performed for numerical purposes, an error is introduced, which depends on $\delta$. In this case, the value of $\delta$ is selected so that the total error does not exceed a given tolerance TOL. See Section 4.5 for more details.

\section{Error analysis}

In this section, we present a simple error analysis to give a qualitative insight into different sources of errors in the presented algorithm. We study the effect of different parameters and factors on the error. The results will be supported by the numerical experiments in the next section.

We consider the micro problem (4) in the local domain $D^{L}$, with

$$
\boldsymbol{\sigma}(\mathbf{u})=\lambda\left(\mathbf{x}, Y^{L}\right) \nabla \cdot \mathbf{u} I+\mu\left(\mathbf{x}, Y^{L}\right)\left(\nabla \mathbf{u}+\nabla \mathbf{u}^{\top}\right) .
$$

The random parameters $\varrho, \lambda$, and $\mu$ are directly obtained from the fiber locations and diameters. The random boundary data $\mathbf{g}^{I}$ in $(4 \mathrm{c})$ is numerically computed and is only an approximation of the true boundary data $\mathbf{g}^{*}$. We assume that $\mathbf{u}^{*}$ is the exact solution to the micro problem (4) with the true boundary data $\mathbf{g}^{*}$. The ultimate goal of computations is to approximate statistical moments of a quantity of interest $\mathcal{Q}\left(\mathbf{u}^{*}\right)$ inside the local domain. For instance, assume that the quantity of interest is a bounded operator applied to the solution, e.g. a component of the stress tensor in the local domain $D^{L}$, and that we want to compute its expected value $\mathbb{E}\left[\mathcal{Q}\left(\mathbf{u}^{*}\right)\right]$. Let $\mathbb{E}_{\ell}\left[\mathcal{Q}_{\delta}\left(\mathbf{u}_{h}\right)\right]$ be the approximation to $\mathbb{E}\left[\mathcal{Q}\left(\mathbf{u}^{*}\right)\right]$, obtained by the algorithm described in Section 3. The total error then reads

$$
\varepsilon:=\left\|\mathbb{E}\left[\mathcal{Q}\left(\mathbf{u}^{*}\right)\right]-\mathbb{E}_{\ell}\left[\mathcal{Q}_{\delta}\left(\mathbf{u}_{h}\right)\right]\right\|
$$

where $\|$. I denotes the $L^{2}\left(0, T ; L^{2}\left(D^{L}\right)\right)$ norm. Note that if the quantity of interest is a functional on the solution, e.g. a spatial and temporal average of the solution over the local domain $D^{L}$, then the norm $\|$. || will simply change to the absolute value.

One can distinguish four different types of errors in the approximation:

1. Error in the calculation of boundary data $\mathbf{g}^{I}$ for the local problem.

2. Spatial and temporal discretization error in the deterministic solver. 


\section{Filtering error.}

4. Error in the stochastic collocation method.

Accordingly, we split the total error into four parts and write

$$
\varepsilon \leq \varepsilon_{I}+\varepsilon_{I I}+\varepsilon_{I I I}+\varepsilon_{I V}
$$

where

$$
\begin{aligned}
\varepsilon_{I} & =\left\|\mathbb{E}\left[\mathcal{Q}\left(\mathbf{u}^{*}\right)\right]-\mathbb{E}[\mathcal{Q}(\mathbf{u})]\right\|, \\
\varepsilon_{I I} & =\left\|\mathbb{E}[\mathcal{Q}(\mathbf{u})]-\mathbb{E}\left[\mathcal{Q}\left(\mathbf{u}_{h}\right)\right]\right\|, \\
\varepsilon_{I I I} & =\left\|\mathbb{E}\left[\mathcal{Q}\left(\mathbf{u}_{h}\right)\right]-\mathbb{E}\left[\mathcal{Q}_{\delta}\left(\mathbf{u}_{h}\right)\right]\right\|, \\
\varepsilon_{I V} & =\left\|\mathbb{E}\left[\mathcal{Q}_{\delta}\left(\mathbf{u}_{h}\right)\right]-\mathbb{E}_{\ell}\left[\mathcal{Q}_{\delta}\left(\mathbf{u}_{h}\right)\right]\right\| .
\end{aligned}
$$

We will consider and discuss each error separately.

\subsection{Error in boundary data}

We first note that due to the well-posedness of the local stochastic IBVP (4), there exists a unique weak solution that depends continuously on the data, see [21]. Consequently, we have for every $Y \in \Gamma$,

$$
\left\|\mathbf{u}^{*}-\mathbf{u}\right\|_{L^{2}\left(0, T, H^{1}\left(D^{L}\right)\right)} \leq C\left\|\mathbf{g}^{*}-\mathbf{g}^{I}\right\|_{L^{2}\left(0, T, H^{1 / 2}\left(\partial D^{L}\right)\right)} .
$$

The magnitude of the first error $\varepsilon_{I}$ is therefore controlled by the error in the approximation of the true boundary data $\mathbf{g}^{*}$.

Next, we note that the error in the boundary data is due to two different sources:

(i) Error due to the pollution effect (discussed in Section 3.2).

(ii) Errors in the computation of the intermediate solution $\mathbf{u}^{I}$, which is in turn due to

- Error in the approximation of the effective stiffness matrix in the meso problem $C^{I}$, including the homogenization error and the truncation error in K-L representation.

- Spatial and temporal discretization error in the meso problem.

- Error in the calculation of the boundary data $\mathbf{g}^{G}$ for the intermediate problem. 
Let $C^{* I}$ be the true stiffness matrix in the meso problem. It is well known that due to the continuous dependence of solution on the coefficients under proper regularity assumptions, one can bound the changes in the solution $\left|\mathbf{u}^{* I}-\mathbf{u}^{I}\right|$ by the changes in the coefficients $\left|C^{* I}-C^{I}\right|$, see e.g. $[11,26]$. For example, in [26], it is shown that if an expansion of the $C^{I}$ coefficients is convergent, then the corresponding solutions will converge to the solution of the limiting system.

\subsection{Discretization error in the deterministic solver}

The discretization error $\varepsilon_{I I}$ in (12) represents the convergence of the deterministic numerical scheme with respect to the mesh size $h$ in the micro problem. With a uniform mesh, we have

$$
\varepsilon_{I I}=\mathcal{O}\left(h^{r}\right), \quad r \geq 1
$$

where $r$ is the minimum between the order of accuracy of the finite element or finite difference method used and the regularity of the solution. We note that both spatial mesh sizes $\Delta x_{1}$ and $\Delta x_{2}$ and time step $\Delta t$ are of order $h$ and related by a proper CFL condition. Also Note that the constant in the term $\mathcal{O}\left(h^{r}\right)$ depends on $\mathcal{Q}\left(\mathbf{u}_{h}\right)$ and is uniform with respect to $Y$, see [22].

\subsection{Filtering error}

Consider a scalar function $Q \in C^{\infty}\left(\mathbb{R}^{2}\right)$. Let $Q_{\delta}(\mathbf{x}):=\left(Q \star K_{\delta}\right)(\mathbf{x})$ be the filtered function, where the kernel and convolutions are defined in (9) and (10). We use the change of variables

$$
\frac{\mathbf{x}-\tilde{\mathbf{x}}}{\delta}=\mathbf{z}
$$

and write

$$
Q_{\delta}(\mathbf{x})=\int_{D^{L}} K_{\delta}(\mathbf{x}-\tilde{\mathbf{x}}) Q(\tilde{\mathbf{x}}) d \tilde{\mathbf{x}}=\frac{1}{2 \pi} \int_{D^{L}} e^{-|\mathbf{z}|^{2} / 2} Q(\mathbf{x}-\delta \mathbf{z}) d \mathbf{z} .
$$

The Taylor expansion of $Q(\mathbf{x}-\delta \mathbf{z})$ around $\mathbf{x}$ gives

$Q_{\delta}(\mathbf{x})=\frac{1}{2 \pi} \int_{D^{L}} e^{-|\mathbf{z}|^{2} / 2}\left(Q(\mathbf{x})-\delta \mathbf{z}^{\top} \nabla Q(\mathbf{x})+\frac{\delta^{2}}{2} \mathbf{z}^{\top} D^{2} Q(\mathbf{x}) \mathbf{z}+\mathcal{O}\left(\delta^{3}\right)\right) d \mathbf{z}$

where, $D^{2}$ is the Hessian matrix. We note that

$$
\int e^{-z_{i}^{2} / 2} z_{i}^{p} d z_{i}=\left\{\begin{array}{ll}
C_{p}, & p \text { even, } \\
0, & p \text { odd },
\end{array} \quad i=1,2,\right.
$$


with $C_{p}$ being a constant. We then obtain

$$
\left|Q(\mathbf{x})-Q_{\delta}(\mathbf{x})\right| \propto \delta^{2}\left|D^{2} Q(\mathbf{x})\right|
$$

where the size of the coefficient $\left|D^{2} Q(\mathbf{x})\right|$ depends on the spatial regularity of $Q$. For instance, when $Q=\mathcal{Q}\left(\mathbf{u}_{h}\right) \in H^{1}\left(D^{L}\right)$, by the inverse inequality, provided a uniform mesh is used in the deterministic solver, we have

$$
\left\|\partial_{x_{i} x_{j}} \mathcal{Q}\left(\mathbf{u}_{h}\right)\right\|_{L^{2}\left(D^{L}\right)} \leq C_{\mathrm{inv}} h^{-1}\left\|\mathcal{Q}\left(\mathbf{u}_{h}\right)\right\|_{H^{1}\left(D^{L}\right)} .
$$

Therefore, we obtain the upper error bound

$$
\varepsilon_{I I I} \leq \mathcal{O}\left(\delta^{2} h^{-1}\right)
$$

As mentioned in Remark 3, the parameters $\delta$ and $h$ are chosen so that the total error does not exceed a given TOL. See Section 4.5 for more details.

\subsection{Error in the stochastic collocation method}

As extensively discussed in $[22,21]$, the error in the stochastic collocation method $\varepsilon_{I V}$ is related to the stochastic regularity of the quantity of interest.

For simplicity, we consider the quantity of interest $\mathcal{Q}(\mathbf{u})=\mathbf{u}$ and investigate the stochastic regularity of $\mathcal{Q}_{\delta}\left(\mathbf{u}_{h}\right)=K_{\delta} \star \mathbf{u}_{h}$. There are two sources of regularity in both spatial and stochastic spaces: the spatial discretization; and the filtering.

We first study the regularity due to the spatial discretization. By employing the inverse inequality, we write for a multi-index $\mathbf{k} \in \mathbb{Z}_{+}^{2}$,

$$
\left\|\partial_{\mathbf{x}}^{\mathbf{k}} \mathbf{u}_{h}\right\|_{L^{2}\left(D^{L}\right)} \leq C_{\mathrm{inv}} h^{-|\mathbf{k}|}\left\|\mathbf{u}_{h}\right\|_{L^{2}\left(D^{L}\right)} .
$$

Through the Stochastic PDE, the spatial regularity can be transformed into the regularity in the stochastic space, see Theorem 6 of [22]. We have

$$
\left\|\partial_{Y}^{\mathbf{k}} \mathbf{u}_{h}\right\|_{L^{2}\left(D^{L}\right)} \leq|\mathbf{k}| ! C^{|\mathbf{k}|}\left\|\mathbf{u}_{h}\right\|_{L^{2}\left(D^{L}\right)}, \quad C=\mathcal{O}\left(h^{-1}\right)
$$

Consequently, for every $Y \in \Gamma$ the power series $u_{h}: \mathbb{C}^{N} \rightarrow L^{\infty}\left(0, T ; H_{0}^{1}(D)\right)$ defined by

$$
u_{h}(t, \mathbf{x}, Z)=\sum_{k=0}^{\infty} \sum_{|\mathbf{k}|=k} \frac{(Z-Y)^{\mathbf{k}}}{\mathbf{k} !} \partial_{Y}^{\mathbf{k}} u_{h}(t, \mathbf{x}, Y)
$$


with $\mathbf{k} !=\Pi_{n=1}^{N}\left(k_{n} !\right)$ and $Y^{\mathbf{k}}=\Pi_{n=1}^{N} Y_{n}^{k_{n}}$ converges for all $Z \in \mathbb{C}^{N}$ such that $\left|Z_{n}-Y_{n}\right| \leq \tau=\mathcal{O}(h)$. By a continuation argument, the function $u_{h}$ can analytically be extended on the whole region

$$
\Sigma(\Gamma, \tau)=\left\{Z \in \mathbb{C}^{N}, \operatorname{dist}\left(\Gamma_{n}, Z_{n}\right) \leq \tau=\mathcal{O}(h), n=1, \ldots, N\right\}
$$

Notice that the radius of the $Y$-analyticity is $\tau=\tau_{h}=\mathcal{O}(h)$.

We next study the regularity due to the Gaussian filtering. Due to the separability of the two-dimensional Gaussian kernel $K_{\delta}$, the spatial derivative of the kernel can be constructed as the product of two one-dimensional Gaussian derivative functions. We then have

$$
\partial_{\mathbf{x}}^{\mathbf{k}} K_{\delta}(\mathbf{x})=\left(\frac{-1}{\sqrt{2} \delta}\right)^{|\mathbf{k}|} H_{k_{1}}\left(\frac{x_{1}}{\sqrt{2} \delta}\right) H_{k_{2}}\left(\frac{x_{2}}{\sqrt{2} \delta}\right) K_{\delta}(\mathbf{x})
$$

where $H_{k}$ is a Hermite polynomial of degree $k$. Hence,

$$
\partial_{\mathbf{x}}^{\mathbf{k}} \mathcal{Q}_{\delta}\left(\mathbf{u}_{h}\right)=\int_{D^{L}} \partial_{\mathbf{x}}^{\mathbf{k}} K_{\delta}(\mathbf{x}-\tilde{\mathbf{x}}) \mathcal{Q}\left(\mathbf{u}_{h}\right)(t, \tilde{\mathbf{x}}, Y) d \tilde{\mathbf{x}}=\mathcal{O}\left(\delta^{-2|\mathbf{k}|}\right) .
$$

As before, the spatial regularity is transformed into the stochastic regularity, and we have

$$
\left\|\left.\partial_{Y}^{\mathbf{k}} \mathcal{Q}_{\delta}\left(\mathbf{u}_{h}\right)\right|_{L^{2}\left(D^{L}\right)} \leq C^{|\mathbf{k}|}\right\| \mathcal{Q}_{\delta}\left(\mathbf{u}_{h}\right) \|_{L^{2}\left(D^{L}\right)}, \quad C=\mathcal{O}\left(\delta^{-2}\right) .
$$

Hence, the radius of the $Y$-analyticity is $\tau=\tau_{\delta}=\mathcal{O}\left(\delta^{2}\right)$.

It is shown in [22] that the interpolation error in the stochastic collocation for a quantity of interest with the radius of $Y$-analyticity $\tau$ is proportional to $e^{-c \tau \ell}$, where $c>0$ is a bounded constant. For example, using an isotropic sparse tensor product interpolation based on Gauss-Legandre abscissas, where $\ell \geq c_{0} / \log N$, with $c_{0}>0$ being a constant, we obtain

$$
\varepsilon_{I V} \leq \mathcal{O}\left(\eta^{-c_{1} \delta^{2} / \log N}\right)
$$

Here $\eta$ is the number of collocation points, which depends on $\ell$, and the type of the sparse grid. Moreover, for any quantity of interest with $s$ bounded mixed $Y$-derivatives, the interpolation error is shown to be proportional to $\eta^{-c_{2} s / \log N}$, where $c_{2}>0$ is a bounded constant [22]. Therefore, we have

$$
\varepsilon_{I V} \leq \min \left(\mathcal{O}\left(\eta^{-c_{1} \delta^{2} / \log N}\right), \mathcal{O}\left(\eta^{-c_{2} s / \log N}\right)\right)
$$


Remark 4. As a simple example, consider the Cauchy problem for the onedimensional acoustic wave equation

$$
\begin{array}{ll}
u_{t t}(t, x, y)-y^{2} u_{x x}=0 & \text { in }[0, T] \times \mathbb{R} \times \Omega, \\
u(0, x, y)=g(x), \quad u_{t}(0, x, y)=0 & \text { on }\{t=0\} \times \mathbb{R} \times \Omega,
\end{array}
$$

By the d'Alembert's formula, the solution reads

$$
u(t, x, y)=\frac{1}{2}[g(x-y t)+g(x+y t)] .
$$

Now, since

$$
\partial_{x} u=\frac{1}{2}\left[g^{\prime}(x-y t)+g^{\prime}(x+y t)\right], \quad \partial_{y} u=\frac{1}{2}\left[-t g^{\prime}(x-y t)+t g^{\prime}(x+y t)\right],
$$

the spatial and stochastic regularity of the solution $u$ are both related to the regularity of $g$ and hence related.

\subsection{Computational cost versus error minimization}

There are three main parameters, $(h, \delta, \eta(\ell))$, that control the computational cost $\mathcal{W}$ and the error $\mathcal{E}:=\varepsilon_{I I}+\varepsilon_{I I I}+\varepsilon_{I V}$. In order to find the optimal choice of the parameters, we need to minimize the computational complexity of the stochastic collocation method, subject to the total error constraint $\mathcal{E}=$ TOL. We first note that

$$
\mathbb{E}_{\ell}\left[\mathcal{Q}_{\delta}\left(\mathbf{u}_{h}\right)\right]=\mathbb{E}_{\ell}\left[K_{\delta} \star \mathcal{Q}\left(\mathbf{u}_{h}\right)\right]=K_{\delta} \star \mathbb{E}_{\ell}\left[\mathcal{Q}\left(\mathbf{u}_{h}\right)\right]
$$

The computational cost of the second term in (16) is of order $\eta\left(\delta^{-d}+h^{-(d+1)}\right)$, while the cost for computing the last term is of order $\delta^{-d}+\eta h^{-(d+1)}$, where $d$ is the spatial dimension (here $d=2$ ). It is therefore more economical to employ the last expression in (16) for computing $\mathbb{E}_{\ell}\left[\mathcal{Q}_{\delta}\left(\mathbf{u}_{h}\right)\right]$. Therefore

$$
\mathcal{W} \propto \delta^{-d}+\eta h^{-(d+1)}
$$

Moreover, from the previous sections, we have for an isotropic sparse tensor product interpolation based on Gauss-Legandre abscissas,

$$
\mathcal{E} \propto h^{r}+\delta^{2} h^{-1}+\eta^{-c \delta^{2} / \log N}
$$

under the assumption that $\varepsilon_{I V}$ is bounded by the first term in (14). 
We now introduce the Lagrange function

$$
\mathcal{L}:=\mathcal{W}+\lambda(\mathcal{E}-\mathrm{TOL})
$$

with the Lagrange multiplier $\lambda$. We note that in practice, the constants in the error terms are important and need to be considered and included in the Lagrange function. The optimal choice of the parameters are then obtained by equating the partial derivatives of the Lagrange functions with respect to the parameters $h, \delta, \eta$, and $\lambda$ to zero.

As an example, consider the case where $\delta$ is fixed. This occurs when for instance the filtered quantity is a physical quantity of interest, see Remark 3. Then the Lagrange function corresponding to (17) and (18) reads

$$
\mathcal{L}=\eta h^{-(d+1)}+\lambda\left(h^{r}+\eta^{-2 / \log N}-\text { TOL }\right) .
$$

The optimal choice of the parameter $h$ is then obtained by

$$
h^{r} \approx \mathrm{TOL} /\left(1+\frac{r \log N}{s(d+1)}\right),
$$

resulting in the computational work $\mathcal{W} \propto \mathrm{TOL}^{-\log N / s-(d+1) / r}$.

\section{Numerical experiments}

In this section, we present several numerical examples to demonstrate the efficiency and applicability of the global-local algorithm described above.

We consider a ply of long unidirectional fibers with zero angle in the state of plane strain in $\mathbb{R}^{3}$. The material is assumed to be isotropic. The governing equation is given by the stochastic PDE (1a) with the stress tensor (2). We assume $\varrho=1$, and let the materials properties, i.e. the Lame parameters $\lambda$ and $\mu$ of fibers and the matrix, be deterministic and given by Table 1 . We further assume that the randomness in the coefficients is only due to the random spatial distribution and random size of fibers. The volume fraction of fibers is assumed to be $59 \%$.

We let $D=[-1500,1500] \times[-600,0]$ be the two-dimensional global domain, which is the orthogonal cross section of the ply, see Fig. 5. We consider homogeneous initial conditions and impose homogeneous Dirichlet (no displacement) boundary condition on the left boundary $\partial D_{1}=\left\{\mathbf{x} \mid x_{1}=\right.$ $\left.-1500, x_{2} \in[-600,0]\right\}$, and homogeneous Neumann (no traction) boundary 


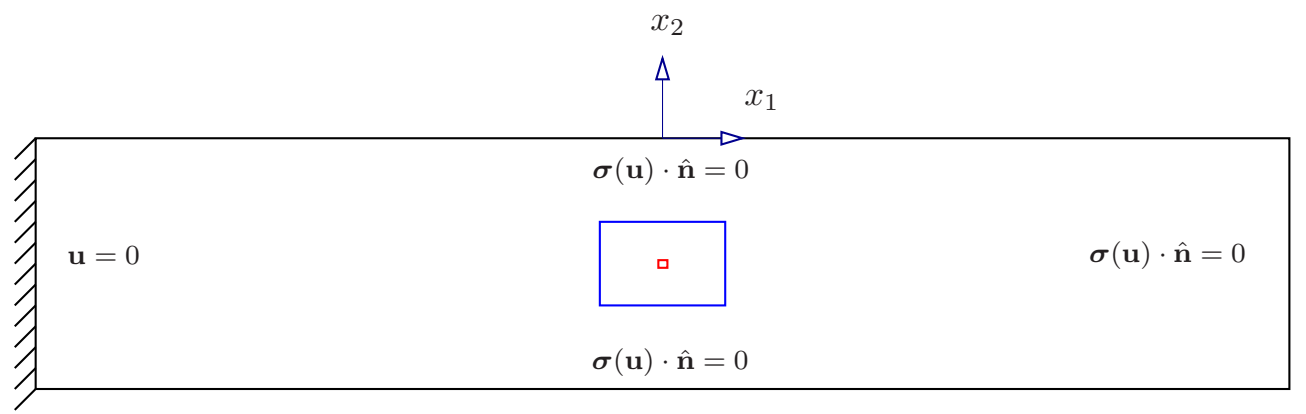

Figure 5: The two-dimensional global domain $D=[-1500,1500] \times[-600,0]$ with homogeneous Dirichlet and Neumann boundary conditions. The small boxes show the intermediate and local domains $D^{I}=[-150,150] \times[-400,-200]$ and $D^{L}$.

condition on $\partial D_{2}=\partial D \backslash \partial D_{1}$. Therefore, we have $\mathbf{g}_{1}=\mathbf{g}_{2}=\mathbf{h}_{d}=\mathbf{h}_{n}=\mathbf{0}$ in (1b)-(1d).

We consider a time-dependent force term $\mathbf{f}=\left(f_{1}, f_{2}\right)^{\top}$ consisting of a Ricker wavelet imposed on the region $D_{f}=[100,200] \times[-350,-250]$,

$$
f_{j}(t, \mathbf{x})=\mathcal{S}(t) \mathbb{I}_{D_{f}}(\mathbf{x}),
$$

where $\mathbb{I}$ is the indicator function, and

$$
\mathcal{S}(t)=0.1\left(1-\omega_{f}\left(t-t_{0}\right)^{2}\right) \exp \left(-\omega_{f}\left(t-t_{0}\right)^{2} / 2\right), \quad \omega_{f}=0.002, \quad t_{0}=10 .
$$

In all examples, we choose a fixed intermediate domain $D^{I}=[-150,150] \times$ $[-400,-200]$. The local domains $D^{L}$ may be different in different examples, but all are contained in $D^{I}$.

The deterministic solver employs an explicit, second order accurate finite difference method which discretizes the PDEs in its second order form [23]. In the stochastic space, we use collocation on the isotropic hyperbolic cross sparse grid based on Gauss abscissas.

\subsection{Example 1}

We first consider a local domain $D^{L}=[-5,5] \times[-302,-298]$ containing only one fiber. We let the fiber diameter be deterministic and fixed $d_{f}=2$. We further let the position of fiber center $\mathbf{x}_{c}$ change only in the horizontal direction and be described by a uniformly distributed random variable:

$$
\mathbf{x}_{c}=\left.\left(x_{1}, x_{2}\right)\right|_{c}=(y,-300), \quad y \sim \mathcal{U}(-3.5,3.5) .
$$


For the meso problem, we choose deterministic parameters $\lambda^{I}=3.9$, and $\mu^{I}=3.7$, which are the mean values given in Table 4 . We therefore have only one uniformly distributed random variable $Y=y$.

Finally, for the macro problem in the global domain $D$, we choose the following deterministic effective parameters

$$
\lambda^{G}=0.59 \lambda_{\text {fiber }}+0.41 \lambda_{\text {matrix }}, \quad \mu^{G}=0.59 \mu_{\text {fiber }}+0.41 \mu_{\text {matrix }} .
$$

We consider the horizontal component of the normal stress as the quantity of interest

$$
\mathcal{Q}(\mathbf{u})=\sigma_{x_{1}}(t, \mathbf{x}, Y) .
$$

We solve the problem using the multi-level algorithm and compute the expected value of the quantity of interest

$$
\mathcal{Q}_{\ell, h}(t, \mathbf{x}):=\mathbb{E}_{\ell}\left[\mathcal{Q}\left(\mathbf{u}_{h}\right)\right](t, \mathbf{x})=\mathbb{E}_{\ell}\left[\sigma_{h x_{1}}\right](t, \mathbf{x}),
$$

where the subscripts $\ell$ and $h$ represent the level in the stochastic space and the mesh size in the micro problem. Fig. 6 shows $\mathcal{Q}_{\ell, h}$ at time $t=70$ and $\mathbf{x} \in D$ with the mesh size $h=0.125$ and for four different levels $\ell=$ $10,20,100,10000$. Since there is only one random variable, the number of collocation points is given by $\eta=\ell+1$.

We observe that for small values of $\ell$, i.e. small number of collocation points, the quantity of interest $\mathcal{Q}_{\ell, h}$ is oscillatory, and as $\ell$ increases the oscillations damp out, and we obtain a uniform solution which is expected. It is however very expensive to use a large number of collocation points, especially in high stochastic dimensions. As an alternative, we propose to use a low number of sample points, which is affordable, and then to perform a low-pass filtering (10) on the oscillatory quantity of interest (19) using a Gaussian kernel (9). Let $\mathcal{Q}_{\ell, h, \delta}:=\mathbb{E}_{\ell}\left[\mathcal{Q}_{\delta}\left(\mathbf{u}_{h}\right)\right](t, \mathbf{x})$, where $\delta$ is the standard deviation of the kernel. We let the non-filtered solution obtained by a large level $\ell^{*}=10000$ be a reference solution and compute two relative errors:

$$
\begin{aligned}
\mathcal{E}_{\infty}(t) & :=\frac{\left\|\mathcal{Q}_{\ell^{*}, h}(t, .)-\mathcal{Q}_{\ell, h, \delta}(t, .)\right\|_{L^{\infty}\left(D_{\delta}\right)}}{\left\|\mathcal{Q}_{\ell^{*}, h}(t, .)\right\|_{L^{\infty}\left(D_{\delta}\right)}}, \\
\mathcal{E}_{2}(t) & :=\frac{\left\|\mathcal{Q}_{\ell^{*}, h}(t, .)-\mathcal{Q}_{\ell, h, \delta}(t, .)\right\|_{L^{2}\left(D_{\delta}\right)}}{\left\|\mathcal{Q}_{\ell^{*}, h}(t, .)\right\|_{L^{2}\left(D_{\delta}\right)}} .
\end{aligned}
$$

We set $D_{\delta}=[-4.25,4.25] \times[-301.25 .-298.75]$. Table 5 shows the relative errors at time $\mathrm{t}=70$ for different levels with a fixed $h=0.125$. We have 


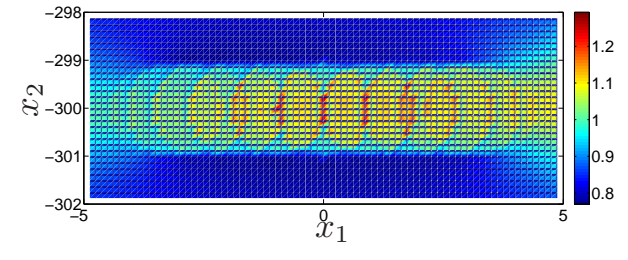

(a) $\ell=10$

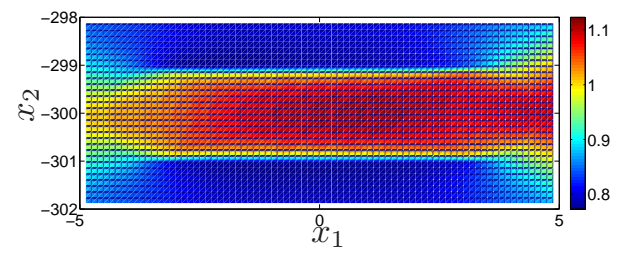

(c) $\ell=100$

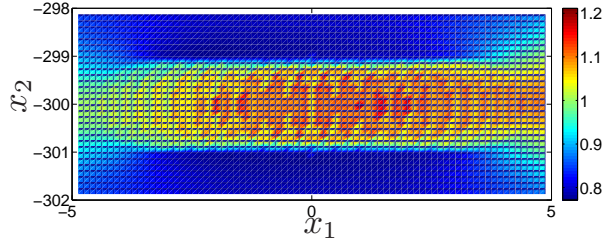

(b) $\ell=20$

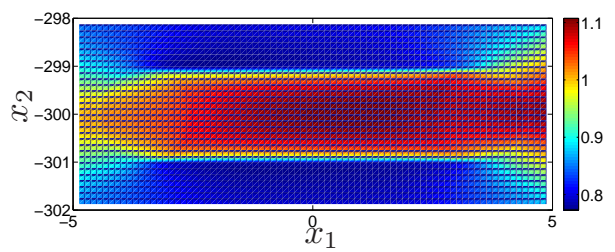

(d) $\ell=10000$

Figure 6: The expected value of the stress $\mathbb{E}_{\ell}\left[\sigma_{h x_{1}}\right](t, \mathbf{x})$ at time $t=70$ and $\mathbf{x} \in D^{L}$, with the mesh size $h=0.125$ and for four different levels $\ell=10,20,100,10000$.

Table 5: Relative errors in the filtered quantity of interest at time $t=70$.

\begin{tabular}{cccc}
\hline levels & $\delta$ & $\mathcal{E}_{\infty}$ & $\mathcal{E}_{2}$ \\
\hline 10 & 1.3 & 0.03 & 0.010 \\
20 & 0.9 & 0.02 & 0.009 \\
100 & 0.4 & 0.01 & 0.003 \\
\hline
\end{tabular}

empirically observed that the choice $\delta=4 / \sqrt{\ell}$ results in small relative errors.

Figure 7 shows the absolute value of the error $\left|\mathcal{Q}_{\ell^{*}, h}(t,)-.\mathcal{Q}_{\ell, h, \delta}(t,).\right|$ in $D_{\delta} \subset D^{L}$ at time $\mathrm{t}=70$ for the level $\ell=20$. Note that since the position of the fiber center changes only in the horizontal direction, we apply filtering only on the horizontal direction in order to avoid introducing errors in the vertical direction, where there are no fibers in the region $x_{2} \in[-302,-301] \cup$ $[-299,-298]$. 


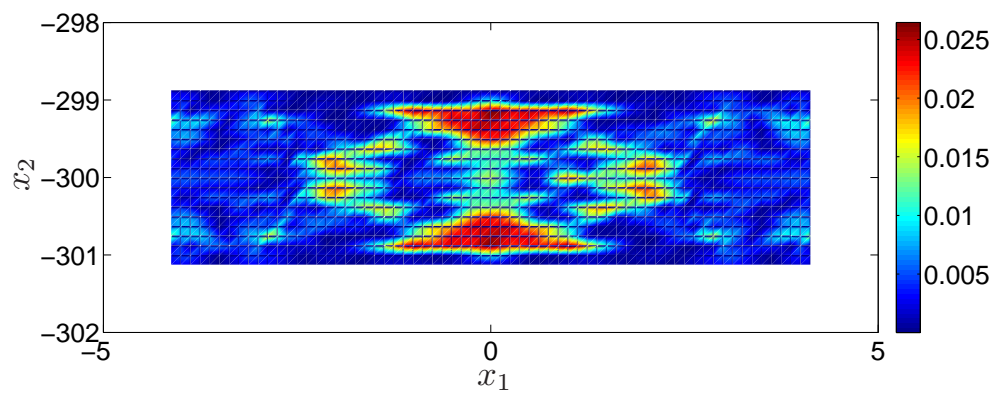

Figure 7: The absolute value of the error $\left|\mathcal{Q}_{\ell^{*}, h}(t,)-.\mathcal{Q}_{\ell, h, \delta}(t,).\right|$ in $D_{\delta} \subset D^{L}$ at time $\mathrm{t}=70$ for the level $\ell=20$ with $\ell^{*}=10000$ and $h=0.125$.

\subsection{Example 2}

We now consider a local domain $D^{L}=[-4,4] \times[-308,-292]$ containing two fibers with deterministic and fixed diameters $d_{f}=2$, see Fig. 8. We further divide the local domain into two equal parts and confine a fiber in each part. This prevents the two fibers from intersecting each other. We let the position of fiber centers change only in the horizontal direction and be described by two independent uniform random variables:

$$
\left.\left(x_{1}, x_{2}\right)\right|_{c_{1}}=\left(y_{1},-300\right),\left.\quad\left(x_{1}, x_{2}\right)\right|_{c_{2}}=\left(y_{2},-300\right),
$$

where

$$
y_{1} \sim \mathcal{U}(-2,-1.05), \quad y_{2} \sim \mathcal{U}(1.05,2) .
$$

This choice will allow the two fibers get very close to each other with a minimum distance of 0.1 .

The material coefficients for the meso and macro problems are deterministic and chosen as in Example 1. We therefore have two independent uniform random variables in total, and hence $Y=\left(y_{1}, y_{2}\right)^{\top}$.

Fig. 9 shows $\mathcal{Q}_{\ell, h}$ in (19) at time $t=70$ and $\mathbf{x} \in D^{L}$ with the mesh size $h=0.1$ and for four different levels $\ell=10,20,1000,5000$ giving $\eta=$ 65, 249, 115889, 1351617 collocation points, respectively. As before, we observe non-physical oscillations for small values of $\ell$, and as $\ell$ increases the oscillations damp out and we obtain a uniform solution as expected. By convolving the stress, obtained by a small level, with a Gaussian kernel, we again obtain good results comparable to the non-filtered stress obtained by a large level. 


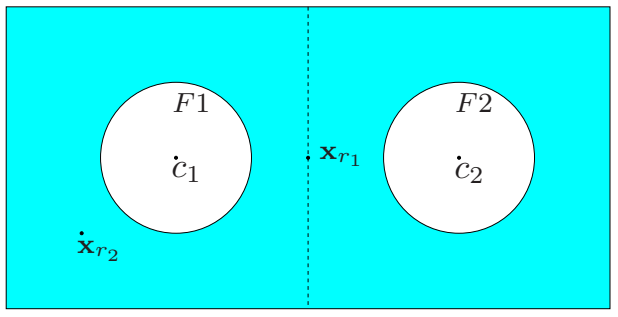

Figure 8: The two-dimensional local domain $D^{L}=[-4,4] \times[-302,-298]$ containing two fibers. The fiber diameters are fixed $d_{f}=2$, and the position of fiber centers are modeled by uniform random variables.

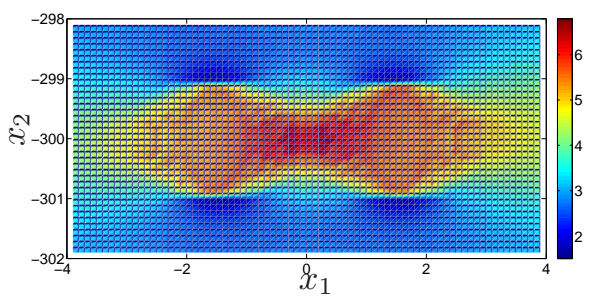

(a) $\ell=10$

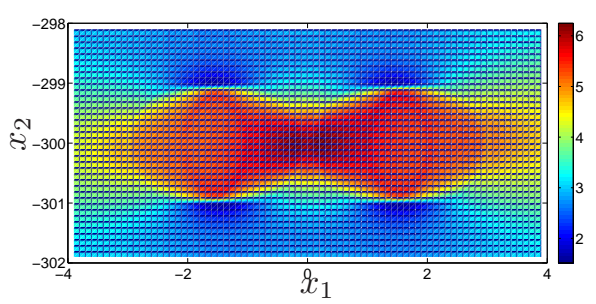

(c) $\ell=1000$

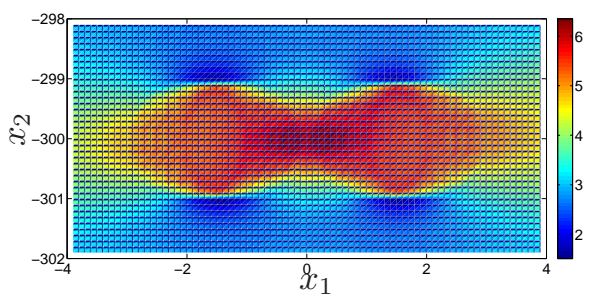

(b) $\ell=20$

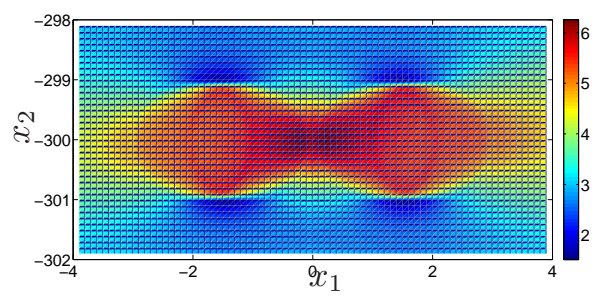

(d) $\ell=5000$

Figure 9: The expected value of the stress $\mathbb{E}_{\ell}\left[\sigma_{h x_{1}}\right](t, \mathbf{x})$ at time $t=70$ and $\mathbf{x} \in D^{L}$ with the mesh size $h=0.1$ and for four different levels $\ell=10,20,1000,5000$.

We next compute the time-history of the expected values of the stress (19) at a point $\mathbf{x}_{r_{1}}=(0,-300)$ between the two fibers. Fig. 10 shows the mean plus and minus the standard deviation of the stress $\sigma_{x_{1}}$ at point $\mathbf{x}_{r_{1}}$ as a function of time.

We also compare the values of stress at two different points: between the two fibers at $\mathbf{x}_{r_{1}}=(0,-300)$, and away from the fibers at $\mathbf{x}_{r_{2}}=(-3,-301)$. Fig. 11 shows that the stress values between two fibers is larger than the stress values at a point away from the fibers. This is due to the fact that the 


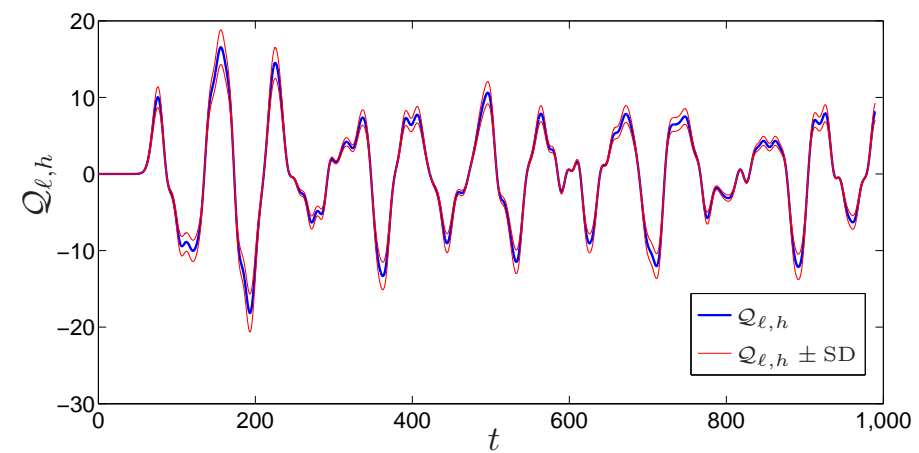

Figure 10: Mean (tick blue line), plus and minus the standard deviation (thin red line) of the stress $\sigma_{x_{1}}$ at point $\mathbf{x}_{r_{1}}$ as a function of time.

fibers can get very close to each other and this concentrates the stress.

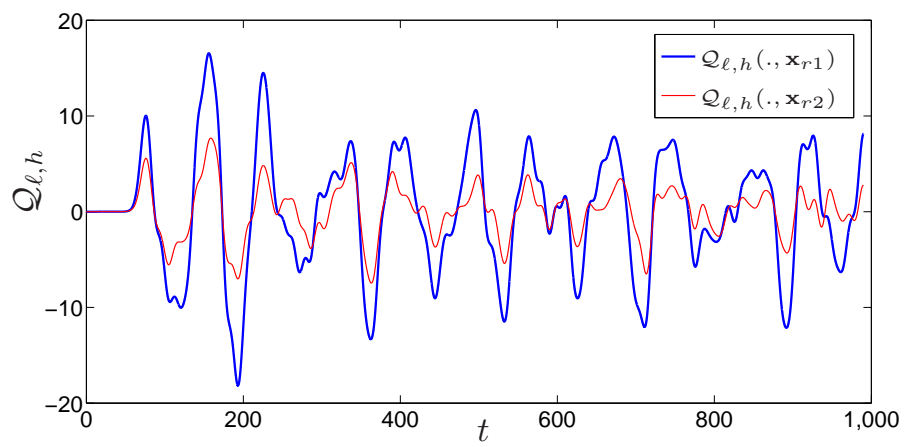

Figure 11: Mean of the stress $\sigma_{x_{1}}$ at two different points $\mathbf{x}_{r_{1}}$ and $\mathbf{x}_{r_{2}}$ as a function of time. Due to the concentration of the stress between fibers, which may be very close to each other, stress at $\mathbf{x}_{r_{1}}$ is higher than stress at $\mathbf{x}_{r_{2}}$.

\subsection{Example 3}

We finally consider a local domain $D^{L}=[-4.5,4.5] \times[-302.5,-297.5]$ containing two fibers with variable diameters. Motivated by [2], we assume that the fiber diameters have a truncated Gaussian distribution with a mean $\mu_{d}=2 \mu \mathrm{m}$ and standard deviation $\sigma_{d}=0.18 \mu \mathrm{m}$ and a cut-off parameter $c_{d}=3$. Therefore, if $y$ is a random variable with a truncated normal density function

$$
\rho_{d}(y)=\frac{\exp \left(-y^{2} / 2\right) \mathbb{I}_{\left[-c_{d}, c_{d}\right]}}{\int_{-c_{d}}^{c_{d}} \exp \left(-z^{2} / 2\right) d z},
$$


the fiber diameters $d_{f_{j}}, j=1,2$, read

$$
d_{f_{j}}=\mu_{d}+\sigma_{d} y_{j}, \quad y_{j} \sim \mathcal{N}_{c_{d}}(0,1), \quad j=1,2 .
$$

We further divide the local domain into two parts and confine one fiber in each part, see Fig. 12. This prevents the two fibers from intersecting each other. We let the position of fiber centers in both horizontal and vertical directions be described by uniformly distributed random variables:

$$
\left.\left(x_{1}, x_{2}\right)\right|_{c_{1}}=\left(y_{3}, y_{4}\right),\left.\quad\left(x_{1}, x_{2}\right)\right|_{c_{2}}=\left(y_{5}, y_{6}\right), \quad y_{j} \sim \mathcal{U}\left(a_{j}, b_{j}\right), \quad j=3, \ldots, 6
$$

where $\left(a_{3}, b_{3}\right)=(-2.3,-1.3),\left(a_{4}, b_{4}\right)=\left(a_{6}, b_{6}\right)=(-300.5,-299.5)$, and $\left(a_{5}, b_{5}\right)=(1.3,2.3)$.

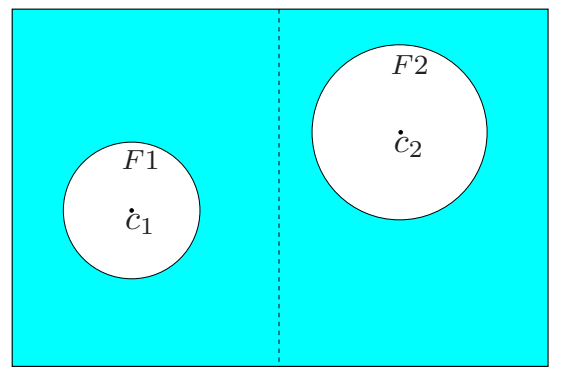

Figure 12: The two-dimensional local domain $D^{L}=[-4.5,4.5] \times[-302.5,-297.5]$ containing two fibers. The fiber diameters and the position of fiber centers are modeled by truncated Gaussian and uniform random variables, respectively.

The stiffness matrix for the micro problem $C^{L}=C^{L}\left(\mathbf{x}, Y^{L}\right)$ is therefore obtained in terms of the random vector $Y^{L}=\left[y_{1}, \ldots, y_{6}\right]^{\top}$ consisting of six independent random variables with truncated Gaussian (two variables) and uniform (four variables) distributions.

For the meso problem in the intermediate domain $D^{I}=[-150,150] \times$ $[-400,-200]$, we use the effective stiffness coefficients obtained as described in Section 3.1 with the correlation length $L_{c}=120$ and the cross-corrolation matrix (3) with $c=0.6$. By choosing $N^{I}=8$ modes in the K-L expansion, $80 \%$ of the standard deviation is preserved. The effective coefficients for the meso problem is therefore obtained in terms of the random vector $Y^{I}=$ $\left[Y_{\lambda}^{I}, Y_{\mu}^{I}\right]$ consisting of $N^{I}=8$ independent normal random variables. Hence, the vector $Y=\left[Y^{L}, Y^{I}\right]$ contains $N=N^{L}+N^{I}=14$ independent random variables. 
The deterministic effective coefficients for the macro problem in the global domain $D=[-1500,1500] \times[-600,0]$, are choosen as in Example 1 .

Fig. 13 shows $\mathcal{Q}_{\ell, h}$ in (19) at time $t=70$ and $\mathrm{x} \in D^{L}$ with the mesh size $h=0.1$ and for two different levels $\ell=10,30$, with the number of collocation points $\eta=6077,218767$, respectively. As before, we observe non-

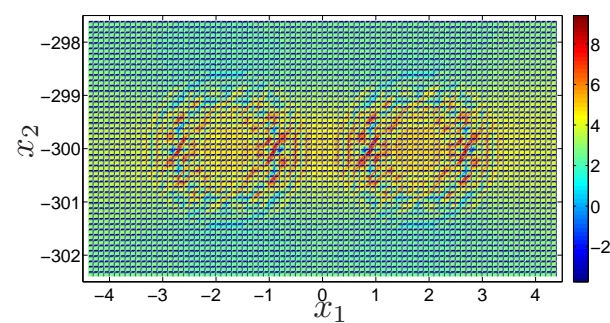

(a) $\ell=10$

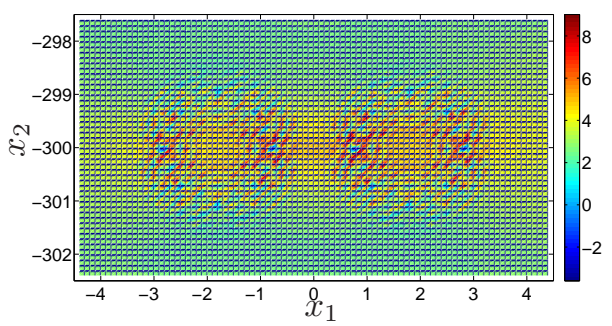

(b) $\ell=30$

Figure 13: The expected value of the stress $\mathbb{E}_{\ell}\left[\sigma_{h x_{1}}\right](t, \mathbf{x})$ at time $t=70$ and $\mathbf{x} \in D^{L}$ with the mesh size $h=0.1$ and for two different levels $\ell=10,30$.

physical oscillations for small values of $\ell$. However, in this case, since the stochastic dimension is relatively large, $N=14$, it is not practical to increase $\ell$ to a high level. Alternatively, we can improve the poor results in Fig. 13 by convolving the solution obtained with a low level $\ell$ with a Gaussian kernel and remove the non-physical oscillations. Fig. 14 shows the filtered quantity $\mathcal{Q}_{\ell, h, \delta}$ with $\ell=10$ and $\delta=4 / \sqrt{\ell}$ in $D_{\delta} \subset D^{L}$ at time $\mathrm{t}=70$ and with $h=0.1$.

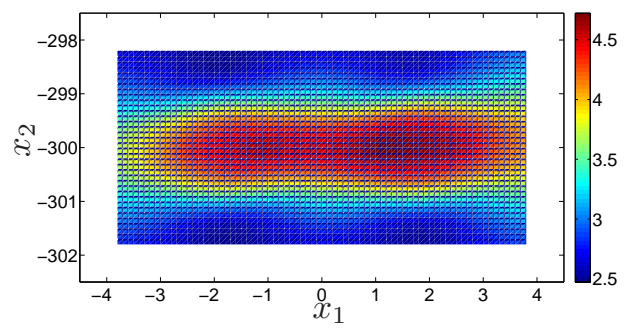

Figure 14: The expected value of the filtered stress $\mathcal{Q}_{\ell, h, \delta}$ with $\ell=10$ and $\delta=4 / \sqrt{\ell}$ in $D_{\delta} \subset D^{L}$ at time $\mathrm{t}=70$ and with $h=0.1$. 
To justify the accuracy of the filtered quantity, we find a reference solution by the Monte Carlo method with $N_{M C}=10^{5}$ sampling points in the stochastic space. Using the obtained reference solution, the relative errors in the filtered quantity $\mathcal{Q}_{\ell, h, \delta}$ with $\ell=10$ at time $t=70$ are $\mathcal{E}_{\infty}=0.04$ and $\mathcal{E}_{2}=0.02$. Hence, using stochastic collocation with only $\eta=6077$ collocation points together with the fast filtering technique, we can obtain accurate solutions, which require $10^{5}$ sampling points if the Monte Carlo method is used.

\section{Conclusions}

We have proposed a stochastic multilevel global-local algorithm for solving the elastodynamic equations in fiber-reinforced composite materials. The method aims at approximating statistical moments of some given quantities of interest, such as stresses, in regions of relatively small size. For a fiberreinforced cross-plied laminate, we introduce three problems (macro, meso, micro) corresponding to the three present scales (laminate, ply, fiber). The algorithm uses the homogenized global solution to construct a good local approximation that captures the microscale features of the real solution. We have presented a qualitative study on different sources of error in the algorithm. We have performed numerical experiments that show the applicability and efficiency of the method. We note that, to the best of the authors' knowledge, the inclusion and propagation of uncertainty in the elastodynamic model for fiber composites is not addressed in the literature, and what we propose in the present work is the first numerical platform for treating such problems.

Future directions include performing a more rigorous error analysis, particularly on the filtering error and interpolation error in the stochastic collocation method, and presenting an alternative numerical algorithm which does not rely on the homogenized global solution. This is the subject of our current work and will be presented elsewhere.

\section{Acknowledgements}

The authors would like to recognize the support of the PECOS center at ICES, The University of Texas at Austin (Project Number 024550, Center for Predictive Computational Science). The first author is supported by the NSF grant DMS 1211014. Supports from the VR project "Effektiva 
numeriska metoder för stokastiska differentialekvationer med tillämpningar" and King Abdullah University of Science and Technology (KAUST) AEA project "Bayesian earthquake source validation for ground motion simulation" are also acknowledged. The third author is a member of the KAUST SRI Center for Uncertainty Quantification in Computational Science and Engineering.

\section{References}

[1] I. Babuska. Homogenization and its applications, mathematical and computational problems. In B. Hubbard, editor, Numerical Solutions of Partial Differential Equations-III, (SYNSPADE 1975, College Park MD, May 1975), pages 89-116, New York, 1976. Academic Press.

[2] I. Babuska, B. Andersson, P. J. Smith, and K. Levin. Damage analysis of fiber composites, Part I: statistical analysis on fiber scale. Comput. Methods Appl. Mech. Engrg., 172:27-77, 1999.

[3] I. Babuska, G. Galoz, and J. Osborn. Special finite element methods for a class of second order elliptic problems with rough coefficients. SIAM J. Numer. Anal., 31:945-981, 1994.

[4] I. Babuska and R. Lipton. $l^{2}$-global to local projection: an approach to multiscale analysis. Math. Mod. Methods Appl. Sci., 21:2211-2226, 2011.

[5] I. Babuska, F. Nobile, and R. Tempone. A stochastic collocation method for elliptic partial differential equations with random input data. SIAM J. Numer. Anal., 45:1005-1034, 2007.

[6] I. Babuska and J. E. Osborn. Generalized finite element methods: Their performance and their relation to the mixed methods. SIAM J. Numer. Anal., 20:510-536, 1983.

[7] G. Bal. Waves in random media. Lecture notes, Columbia University, NY, 2006.

[8] J. F. Bourgat. Numerical experiments of the homogenization method for operators with periodic coefficients, volume 707 of Lecture Notes in Mathematics. Springer-Verlag, 1977. 
[9] A. Bourgeat and A. Piatnitski. Approximation of effective coefficients in stochastic homogenization. Ann. Inst. H. Poincare Prob. Statistics, 40:153-165, 2004.

[10] J. Byström, J. Engström, and P. Wall. Periodic approximation of elastic properties of random media. Advances in Algebra and Analysis, 1:103$113,2006$.

[11] J. Charrier. Strong and weak error estimates for elliptic partial differential equations with random coefficients. IMA J. Numer. Anal., 50:216-246, 2012.

[12] W. E and B. Engquist. The heterogeneous multiscale methods. Commun. Math. Sci., 1:87-132, 2003.

[13] W. E, B. Engquist, X. Li, W. Ren, and E. Vanden-Eijnden. Heterogeneous multiscale methods: A review. Commun. Comput. Phys., 2:367450, 2007.

[14] Y. Efendiev and T. Y. Hou. Multiscale finite element methods. Springer.

[15] B. Engquist, H. Holst, and O. Runborg. Multiscale methods for wave propagation in heterogeneous media. Commun. Math. Sci., 9:33-56, 2011.

[16] B. Engquist and P. E. Souganidis. Asymptotic and numerical homogenization. Acta Numer., 17:147-190, 2008.

[17] T. Y. Hou and X. Wu. A multiscale finite element method for elliptic problems in composite materials and porous media. J. Comput. Phys., 134:169-189, 1997.

[18] T. J. R. Hughes, G. R. Feijoo, L. Mazzei, and J. B. Quincy. The variational multiscale method. A paradigm for computationnal mechanics. Comput. Methods Appl. Mech. Engrg., 166:3-24, 1998.

[19] W. F. Krajewski and C. J. Duffy. Estimation of correlation structure for a homogeneous isotropic random field: A simulation study. Computers \& Geosciences, 14:113-122, 1988.

[20] V. Miroslav. Simulation of simply cross correlated random fields by series expansion methods. Structural Safety, 30:337-363, 2008. 
[21] M. Motamed, F. Nobile, and R. Tempone. Analysis and computation of the elastic wave equation with random coefficients. IMA J. Numer. Anal., 2012. (under review).

[22] M. Motamed, F. Nobile, and R. Tempone. A stochastic collocation method for the second order wave equation with a discontinuous random speed. Numer. Math., 2012. (to appear).

[23] S. Nilsson, N. A. Petersson, B. Sjögren, and H.-O. Kreiss. Stable difference approximations for the elastic wave equation in second order formulation. SIAM J. Numer. Anal., 45:1902-1936, 2007.

[24] J. T. Oden and K. S. Vemaganti. Estimation of local modeling error and goal-oriented adaptive modeling of heterogeneous materials. I: error estimates and adaptive algorithms. J. Comput. Phys., 164:22-47, 2000.

[25] S. A. Smolyak. Quadrature and interpolation formulas for tensor products of certain classes of functions. Doklady Akademii Nauk SSSR, 4:240-243, 1963.

[26] C. C. Stolk. On the modeling and inversion of seismic data. PhD thesis, Utrecht University, The Netherlands, 2000.

[27] T. Strouboulis, I. Babuska, and K. Copps. The design and analysis of the generalized finite element method. Comput. Methods Appl. Mech. Engrg., 192:3109-3161, 2003.

[28] K. S. Vemaganti and J. T. Oden. Estimation of local modeling error and goal-oriented adaptive modeling of heterogeneous materials. II: a computational environment for adaptive modeling of heterogeneous elastic solids. Comput. Methods Appl. Mech. Engrg., 190:6089-6124, 2001.

[29] D. Xiu and J. S. Hesthaven. High-order collocation methods for differential equations with random inputs. SIAM J. Sci. Comput., 27:11181139, 2005. 